\title{
Electrodeposition of Iron-Group Alloys into Nanostructured Oxide Membranes: Synthetic Challenges and Properties
}

\author{
Henrikas Cesiulis ${ }^{1, *}$, Natalia Tsytsaru ${ }^{1,2, *}$, Elizabeth J. Podlaha ${ }^{3}$, Deyang $\mathrm{Li}^{4}$ and Jordi Sort ${ }^{5,6}$ \\ ${ }^{I}$ Department of Physical Chemistry, Vilnius University, Vilnius, LT-03225, Lithuania; ${ }^{2}$ Institute of Applied Physics of \\ ASM, Chisinau, MD-2028, Moldova; ${ }^{3}$ Department of Chemical and Biomolecular Engineering, Clarkson University, \\ Potsdam, New York 13699, USA; ${ }^{4}$ Department of Chemical Engineering, Northeastern University, Boston, Massachu- \\ setts 02115, USA; ${ }^{5}$ Physics Department, Universitat Autònoma de Barcelona, E-08193 Bellaterra, Spain; ${ }^{6}$ Institució \\ Catalana de Recerca i Estudis Avançats (ICREA), Pg. Lluís Companys 23, E-08010 Barcelona, Spain
}

\begin{abstract}
Background: Quasi-one dimensional nanostructures: nanowires, nanotubes, nanorods, nanobelts/nanoribbons and complex "nanowire-nanoparticle" composites have been synthesized over the years. These nanostructures are particularly appealing due to their specific properties defined by their high aspect ratio: two dimensions are in the nanoscale range and one dimension is in the microscale.

Methods: One of the well-designed approaches for the synthesis of such nanostructured materials is template-assisted fabrication combined with electrodeposition. The fabrication approaches for the growth of iron-group alloy nanostructures inside nanoporous oxide membranes by means of different electrodeposition techniques, and the resulting unique properties and potential applications of this type of materials are reviewed.

Results: Arrays of nanostructures can be obtained by filling a porous oxide template that contains a large number of straight cylindrical, nano-sized diameter holes. Generalities of metals electrodeposition into nanoporous oxide membranes are discussed. Measures to minimize the nonuniformity of deposits inside pores need to be addressed to thin the barrier layer, to control hydrogen evolution and to improve mass transport inside the pores. Examples of binary and ternary iron group alloys grown inside nanoporous oxide templates are provided. Catalytic hydrogen evolution and methanol oxidation on the nanowires arrays are described. The "sample size effect" on the magnetic properties of materials and the electrodeposition of multilayered structures necessary for giant magnetoresistance (GMR) are discussed in details.
\end{abstract}

Conclusion: Electrodeposition of binary and ternary iron-group alloys confirm that controlling alloy composition inside nanopores is still a challenge.

Keywords: Electrodeposition, barrier layer, iron-group alloys, nanowires, mass transport, catalytic properties, magnetic properties.

\section{INTRODUCTION}

Nanostructured materials can have versatile technological applications, including areas such as drug delivery and device technology; in turn, they also attract fundamental interest due to substantial changes at the transition from macroto nanoscale [1]. Quasi-one-dimensional nanostructures such as nanowires [2], nanotubes [3, 4], nanorods [5], nanobelts or nanoribbons [6] and complex "nanowire-nanoparticle"

*Address correspondence to these authors at the Institute of Applied Physics of ASM, Chisinau, MD-2028, Moldova, Tel: +373(22) 738178;

E-mail: ashra_nt@yahoo.com

Department of Physical Chemistry, Vilnius University, Vilnius, LT-03225, Lithuania, Tel: +370 (5) 2193178; E-mail: henrikas.cesiulis@chf.vu.lt composites [7] have been synthesized over the years. These nanostructures are particularly appealing due to their specific properties defined by their high aspect ratio: two dimensions are in the nanoscale range and one dimension is in the microscale. One of the well-designed approaches for the synthesis of such nanostructured materials is template-assisted fabrication combined with electrodeposition, first reported by Possin [8] in track etched mica films, and expanded upon by others with different materials and templates [2, 9-14]. However, the self-organized porous anodic aluminum oxide (AAO) membrane has become one of the most often used templates for tunable synthesis of different nanostructured materials $[15,16]$. 
Many end applications require nanostructures with high aspect ratio and/or specific surface area, production reproducibility, uniformity in wire size and shape, and high spatial ordering. Recently, the procedures to tune pore size and pitch distance in AAO have been designed, e.g., [17-20]. The mechanical properties of the membrane are also of importance. It was shown [21] that Young's modulus of AAO depends on the porosity level and the anodizing bath components. For a given porosity, oxalic acid anodization produces an oxide layer with higher elastic modulus as compared to samples prepared from a sulphur-based electrolyte.

The chemical composition of alumina films differs depending on the composition of the anodizing solution as well as anodizing conditions due to the incorporation of acid anions and water molecules into the outer part of alumina cells. For example, the sulfuric acid alumina films contain high amounts of anionic species (12-14 wt.\% sulfate), while the phosphate and oxalate contents in corresponding alumina films are 6-8 and 2-4 wt.\%, respectively [22]. Incorporated anion species produce a negative surface charge of the pore walls influencing the pore filling process by one or another material. The hydrophobic/hydrophilic pore wall properties also play a significant role in nanowire (nanotube) growth in the precursor solution. The roughness and pore length induced wettability was systematically examined for oxide films grown by a two-step, high-field anodization in phosphoric acid [23].

Arrays of nanowires can be obtained by filling a porous template that contains a large number of straight cylindrical, nano-size diameter holes, by various techniques including electrodeposition [15], chemical vapor deposition [24], FIBinduced deposition [25], or molecular beam epitaxy [26]. Among all of these approaches, electrodeposition has been widely applied for the fabrication of nanostructures at different length scales, including the nanometric regime. A requirement for electrodeposition is that a current collector needs to be present, which is made by coating one side of the nanoporous template with a metal film, which serves as a cathode [27]. Nanometric structural features of nanocrystalline metallic systems relevant to the functional properties are grain size, regions of the material exhibiting a given orientation of the crystal lattice (i.e., crystallographic texture), atomic density gradients and zones with a different chemical composition [28]. However, electrodeposition typically operates far from equilibrium conditions [29]. Consequently, the material obtained can show a non-equilibrium structure, which can manifest as small grain sizes and the associated large volume fraction of grain boundaries and triple junctions, as well as non-equilibrium phases. In addition, alloys produced by this method can show considerable extensions of the solid solubility range similar to what is observed in materials produced by other non-equilibrium processing routes, such as rapid solidification. For example, electrodeposited Ni-P can form solid solutions containing phosphorus levels of $10 \mathrm{wt} . \%$ or more $[29,30]$. Similarly, extended solubility ranges were also observed in other alloys, such as CoW [31], Zn-Ni [32], Ni-Mo [33], Fe-Pd [34]. Moreover, new phases may form in nanostructured alloys fabricated by electrodeposition; for example, highly textured thin films of nonstoichiometric $\mathrm{NiWO}_{4}$ were formed during co-deposition of nickel and tungsten [35].
Furthermore, nanostructured materials based on irongroup alloys with diameters about, or smaller than $100 \mathrm{~nm}$, can result in interesting properties. Thus, ferromagnetic $(\mathrm{Fe}$, $\mathrm{Co}, \mathrm{Ni}$ and related alloys) nanowires and nanotubes have gained attention recently due to the ability to tailor their magnetic properties [36-40], having potential applications in the fields of magneto-electronics [41], high sensitive giant magnetoresistance (GMR) sensors [42, 43] or catalysis [44]. It has been found that coercivity, saturation magnetization, and remnant magnetization are strongly dependent on the size, morphology, crystalline structure and composition of nanowires $[45,46]$. Therefore, patterned magnetic storage media has attracted sufficient attention because of its promising potential applications in ultra-high-density storage media $[47,48]$. The recording units of patterned storage media have uniform sizes, shapes and are distributed to overcome the superparamagnetic limit of traditional patterned magnetic recording media at room temperature, while enhancing the signal-to-noise ratio, to achieve recording densities beyond $100 \mathrm{Gbit} / \mathrm{in}^{2}$ [49].

After filling pores with the metal, the templates can be used for various purposes as a composite material (metal/dielectric, metal semiconductor). Alternatively, the formed metal nanostructures can also be released from the template by dissolution and be used for widespread purposes. Nanomaterials, compared with their conventional bulk counterparts, possess relatively high specific surface area and surface atoms. The nanowires released from the template can be deposited onto carbon or another substrate and used, for example, for electroanalytical purposes, as reviewed in [50]. Since the diameters of nanowires are small and comparable to the size of biological and chemical species being sensed, their properties can be influenced by the adsorption of certain analytes. Therefore, magnetic nanowires are increasingly attractive in particular for biological and biomedical applications [51]. These characteristics render them superior electrocatalytic activity compared to other materials with larger lateral sizes. For electrode materials in hydrogen evolution reactions, the enhancement of their activities by adopting nanomaterial fabricated inside $\mathrm{AAO}$ has been frequently employed $[52,53]$.

Thus, fabrication of metallic nanostructured materials is required in catalysis, sensing, electronics, energy harvesting and storage, and applications of materials with tailored magnetic and optical properties. The aim of the present review is to address iron-group alloys nanostructures uniquely, fabricated within oxide membranes by means of different electrodeposition techniques, and to summarize their unique properties and potential applications.

\section{GENERALITIES OF METALS ELECTRODEPOSI- TION INTO NANOPOROUS OXIDE MEMBRANES}

There are three types of oxide membrane/conductive cathode distinguished [46]:

(i) Deposition into free-standing membranes. A sufficiently thick alumina film is formed on Al foil and then released to obtain a self-standing membrane with through pores using sequential chemical etching of the non-oxidized Al. Physical vapor deposition of a thin metal film on one of the membrane surfaces completes the process of obtaining 
the desired sample geometry to begin electrodeposition process. Since it is difficult to obtain thick, self-standing AAO films with small pores, this method is not suitable for the synthesis of metallic nanowires (nanotubes) having small diameters.

(ii) Deposition into on-substrate anodic alumina/Al templates. In this case, the AAO layer is kept on the Al substrate (Fig. 1). As an advantage of this method, both thin and thick on-substrate $\mathrm{AAO} / \mathrm{Al}$ templates can be used to prepare metallic nanowires, thus permitting access even to the sub-20 $\mathrm{nm}$ (pore/wire diameter) range.

(iii) Deposition into supported thin film AAO/metal/substrate (Fig. 2). AAO films are obtained through anodization of a thin Al foil that is fixed onto conductive or metal-coated substrates. Together with sputter deposition or chemical vapour deposition of $\mathrm{Al}$, unique substrate shapes can be coated, combined with very thin or even the absence of a barrier layer. Major benefits of this method are: it allows deposition under DC conditions, is applicable to high ex-situ annealing temperatures, and free-standing metal nanowires (MNW) arrays are obtained by subsequent etching of the $\mathrm{Al}_{2} \mathrm{O}_{3}$ template [54]. The drawbacks are: a) costly high vacuum deposition is required for the growth of Al films; b) several metallic interfacial layers may be needed, which typically requires an inert $(\mathrm{Au}$ or $\mathrm{Pt})$, and buffer layers $(\mathrm{Ti}, \mathrm{Nb}$, etc.) deposited to improve adhesion and prevent substrate oxidation during anodization of aluminium.

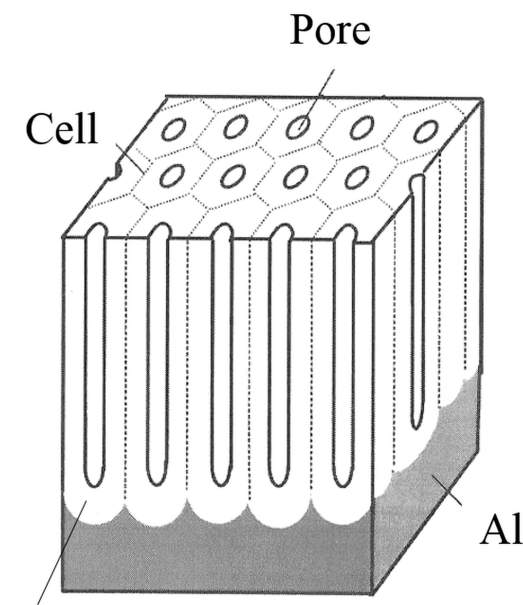

Barrier layer

Fig. (1). Schematic structure of porous-type alumina film onto aluminium.

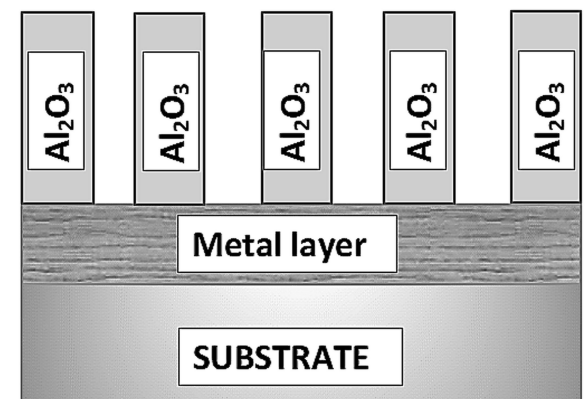

Fig. (2). Schematic structure of the system: AAO/metal/substrate.
In order to be useful, these devices require uniform nanostructure (e.g., nanowires, nanotubes) deposition and repeatable results, fabrication on a large area, high-density arrays of high-aspect-ratio ferromagnetic nanowire structures. These requirements can be challenging. Non-uniform nanowire growth in porous templates is often observed and the plausible reasons for this were identified [55]; namely, variations in interpore spacing in the commercial templates, hydrogen gas evolution blocking some of the pores, nonuniformities in nucleation rates from pore-to-pore, nonuniform mass transfer inside pores. Improvement of uniformity of templates and mass transport as well as control of hydrogen evolution can improve the uniformity of nanowire growth.

\subsection{Barrier Layer Challenges During Electrodeposition into On-substrate Anodic Alumina/Al Templates}

Frequently, lab-made membranes of AAO are fabricated under procedures allowing to receive ordered pores as depicted in Fig. (1), where the size of the pores can be well controlled within micrometer and nanometer scales by careful selection of the processing conditions (e.g., temperature, voltage, type of electrolyte and electrolyte concentration, etc.). Then, the obtained membranes can be used in downstream applications. However, the formation of a nonconductive barrier layer closing the pore bottom and hindering the direct electrochemical growth of metallic nanostructures within the template is a problem (Fig. 1), because the insulating properties of the barrier layer significantly affect the uniformity and quality of the depositing material [56]. The thinning of the barrier layer is performed after the last aluminium anodization stage $\left(1^{\text {st }}\right.$ or $2^{\text {nd }}$, dependently on the anodization procedure applied). Several strategies have been developed to remove the barrier layer [57-61], but none of them allows using AAO readily as produced without undertaking the meticulous procedures or requiring expensive laboratory equipment. Few relatively simple treatments were explored in order to perform dissolution of the nonconductive barrier oxide layer of anodic aluminium nanotemplate [62]: in a zincate solution, or directly in the acidic anodization solution containing $\mathrm{CoCl}_{2}$. The experiments carried out reveal that anodic treatment in the zincate electrolyte remains the best option for barrier oxide layer removing. One optimal result, with a minimum pore enlargement versus maximum barrier layer removal, is obtained at $30^{\circ} \mathrm{C}$, under ultrasonic conditions after $40 \mathrm{sec}$ immersion time [63]: after the zincate treatment, AAO films remain intact on the base aluminium metal and can be readily used as template for further applications without requiring any other additional processes (see Fig. 3), and it was proven by the DC electrodeposition of nickel nanowires. In addition, it is documented that $\mathrm{Co}-\mathrm{Ni}$ binary alloy nanowires of different compositions were co-deposited in the nanopores of highly ordered AAO templates from a single sulphate bath using alternating current (AC) electrodeposition without modifying or removing the barrier layer [64]. 


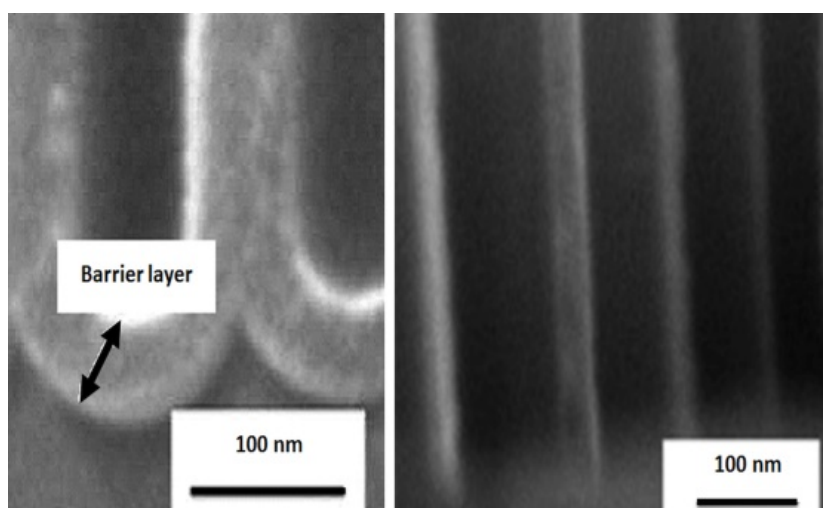

Fig. (3). AAO membrane cross-section before (left image) and after zincate treatment (right image).

\subsection{Hydrogen Evolution from the Nanopores}

Another challenge is that during electrodeposition of iron group metals and their alloys from an aqueous solution, a hydrogen evolution side reaction occurs. In template-assisted electrodeposition into nanopores, gas bubbles from the side reaction can block pores inhibiting or blocking the growth of metal nanowires. Moreover, as $\mathrm{H}_{2}$ evolves nearby the electrode, the local $\mathrm{pH}$ can be altered, which can affect the metal deposition mechanism. For alloys, this leads to changes in composition, as well as in the extreme case of exceedingly increasing $\mathrm{pH}$, this favours the formation of intermediate and low soluble hydroxo-compounds of iron group metals. For minimization of $\mathrm{pH}$ changes, the buffering additives to electrolytes such as boric acid [65], $\mathrm{Na}_{2} \mathrm{CO}_{3}$ [66], ammonia and ammonium compounds $[67,68]$ are used. Also, these additives are the determining conditions to minimize the rate of the hydrogen evolution reaction and to maximize current efficiency [69].

One strategy to manage the side reaction is to use pulse current electrodeposition with long, relaxation or off-times to allow for the diffusion of ions and gas into and out of nanopores. This point of view may be illustrated by successful pulse deposition of Super Invar (Fe-Ni-Co) nanowires [70].

On the other hand, hydrogen bubble generated by the side reaction is a key factor in the fabrication of tubular nanostructure (nanotubes). It was found that the thickness of the nanotube wall could be controlled by adjusting the electrolyte $\mathrm{pH}$ and cathodic potential [71]. The nanotube inner diameter was correlated with the size of the hydrogen bubbles trapped inside the center of the nanopore. Under certain conditions, it is possible to deposit from the same electrolyte either nanowires (pulsed current mode) or nanotubes (direct current mode) [72]. Motoyama et al. [73] elegantly showed a mapping of nickel wires and tubes dependent upon the applied potential and electrolyte $\mathrm{pH}$, and also indicated a transition region where tubes start to grow and then become wires, due to a change of the relative rates of metal and side reaction. Their results suggest that the nanotube deposition starts to occur as the ratio of the metal deposition rate to the hydrogen evolution rate decreases. Davis and Podlaha [74] electrodeposited $\mathrm{Cu}$ and $\mathrm{CoNiCu}$ nanotubes in AAO templates having a contact layer on its backside (free-standing gold sputtered membrane). They used a dilute metal ion electrolyte designed purposely to have a high side reaction and low current efficiency that was deemed critical to the evolution of tube formation. Both gas evolution, resulting from proton and water reduction and partial pore coverage on the bottom of the membranes accounted for the tube formation. By only increasing the current efficiency, wires were formed. In contrast, lowering the current efficiency resulted in tubes. Others have reported electrodeposited tubes in nanoporous membranes. For example, in the case of $\mathrm{Cu}$ [75] and $\mathrm{Ni}$ tubes [76] electrodeposition, the formation of a tubular structure was explained exclusively by the partial pore filling of the substrate by the conductive Au layer, created on the backside of the AAO. A small amount of Au coats the inner walls at one of the membranes, not entirely filling up the pore end. Thus, the conductive region protruding into the pore along the sidewall and near the bottom of the pore creates a non-uniform electric field, promoting deposition along the sidewall resulting in the formation of tubes. Davis and Podlaha [74] noted that tube formation is also dependent on mass transfer controlled deposition and hydrogen evolution that was deemed essential to promote tube formation of $\mathrm{Cu}$ and $\mathrm{CoNiCu}$ nanotubes.

Recognizing that the side reaction plays an important role in determining the deposit morphology, Philippe and Michler [77] presented a model that predicts nanostructure geometry (tubular versus nanowire) of metals depending on the electrolyte $\mathrm{pH}$, applied potential, deposition time and the pore density, length and radius. The model assumes a diffusion controlled metal reduction reaction that is under transport control and $\mathrm{pH}$ dependent. For higher $\mathrm{pH}$ values, the metal thickness at the wall approaches the pore radius and the structure is a wire. This model was successful at predicting the relative tube thickness for electrodeposited cobalt wires.

Fukunaka et al. [71] described the role of hydrogen bubbles when tubes are formed in nanoporous membranes. During deposition, a gas bubble remains in the core blocking the metal deposition. The gas generation is balanced with its dissolution rate, forming soluble hydrogen gas molecules, dictated by Henry's law, at the front face of the trapped bubble. Further bubble nucleate can occur outside of the pores when the hydrogen's critical concentration is reached and can affect the hydrodynamic environment near the pore mouth.

\subsection{Uniformity and Mass Transport}

The criteria for successful electrodeposition in the recesses are the void-free and uniform filling of them in a timely manner [78]. Nonuniform ion transport outside and inside the template pores result in the non-uniform growth of the nanowires. This, in turn, creates only a few nanowires that fill the pore from one side of the template to another. These nanowires start to create semi-spherical caps that completely block the other remaining pores having shorter nanowires, especially in widely used porous anodic aluminium oxide (AAO) due to its high porosity and packing density. This is extremely critical in fabricating the nanowire-based devices since only limited numbers of the nanowires are actually in contact at both ends. In other words, a major portion of the nanowires is malfunctioning [79]. 
Mass transport is of critical importance during electroforming, and the presence of an array of cylindrical pores on the electrode has a significant effect on the diffusion [80]. The different concentration profiles result in different wire growth, and it is attributed to the overlapping of diffusion regions, which results in less diffusive flux in certain pores and more diffusive flux in other pores resulting in nonuniform nanowire lengths [81]. Methods to increase masstransport of the deposited metal ions include ultrasonic agitation [82], increasing the temperature of the electrolyte [83], and pulse-electrodeposition modalities [84]. With increasing pore lengths and decreasing in radial dimensions, a lower contribution of convection to mass transport can be observed [85]. However, Amatore et al. [86] showed that even when an electrolyte is macroscopically still, there can be a microscopically chaotic motion that can be viewed as an apparent diffusion coefficient that depends on the position and is particularly useful at long transient. Given the consumption of ions within pores, a natural convection can result. For example, Konishi [87] showed that there was the difference in the growth rate of copper wires, as reflected in the current transient if the cathode was placed facing up or down. With the cathode up, mass transport fluxes are aided by both diffusion and natural convection while the opposite orientation shows a lower current transient as only diffusive fluxes are present.

The transient diffusion problem with two fixed boundary layers can be readily solved analytically, for an array of recessed electrodes, such as the prediction of the transient current in Eq. (1), for a constant potential with a single metal reactant that describes linear diffusion in a pore [88]:

$$
I=n F\left(\frac{D}{\pi r_{0}}\right)^{\frac{1}{2}}\left(C_{b}-C_{m}\right)\left\{1-\exp \left[\frac{4 \pi N r_{0}^{2} C_{b} \sqrt{\pi t D}}{\left(4 L+\pi r_{0}\right)\left(C_{b}-C_{m}\right)}\right]\right\}
$$

The equation does not assume that the deposited film is growing and so is only valid at the very start of deposition.

Mass-transfer controlled metal reactions inside the pores of the AAO template were investigated-and a simplified model was proposed [89]. When a metal is deposited inside nano- or mesopores, natural convection inside the pores had not be taken into account ${ }^{1}$ at first approximation due to the limited cross-section and essentially shorter pore length than the length of the layer of convective diffusion, and metal ions are supplied by linear diffusion. The highest rate of diffusion occurs when the concentration of reducing metal ions at the bottom of the pores with the length $l$ is equal to zero. In this case, diffusion undergoes in accordance with the second Fick's law for the planar diffusion. An estimate of the time it takes for diffusion from the bulk of solution toward the pores takes place and finally a stationary flux of metal ions is realized, where a non-stationary diffusion regime is switched to the stationary after time $t_{t r}$ :

$$
t_{t r}=\frac{L^{2}}{D \pi}
$$

\footnotetext{
${ }^{1}$ Usually, the electroplating baths contains the salts of depositing metals and ligands, buffering agents as well at concentrations comparable or even higher that metal concentration, therefore, electrolyte density variations due to metal ions electroreduction is negligible small.
}

Where, $L$ is a pore length, $D$ is a diffusion coefficient of reducing metal ions

At this point, it should be clarified that diffusion is spherical at the top of the pores ('outer layer') because of the sub-micron size of the diffusion plane, and remains planar inside the AAO pores (see Fig. 4a). Therefore, the 'outer' diffusion layer is likely to be very thin in comparison with a planar diffusion layer, otherwise a planar diffusion only unable to support metal transport to the electrode at the relatively high current density (3-5 $\mathrm{mA} \mathrm{cm}^{-2}$ in the case of Co$\mathrm{W})$. Therefore, for the electrodeposition of Co-W alloys in the nanosized recesses is governed by the diffusion of discharging metal-containing species from the bulk solution toward the pores, while the electrodeposition on flat electrodes occurs under kinetic limitations [90].

The more comprehensive models were presented by Blanco et al. [91] and Philippe et al. [92], that takes into account the growing metal film in the pores and as a result a changing metal ion concentration at the pore mouth with a constant bulk concentration. The model assumes that at the start of deposition, the diffusion, and thus the concentration gradient changes in one dimension along the pore; a transition occurs as the front reaches the pore mouth and then creates a three-dimensional hemispherical front. Eventually, the neighboring boundary layers near the pore mouth start to overlap and mass transport can be described as planar diffusion to the whole array like it is shown in Fig (4b).

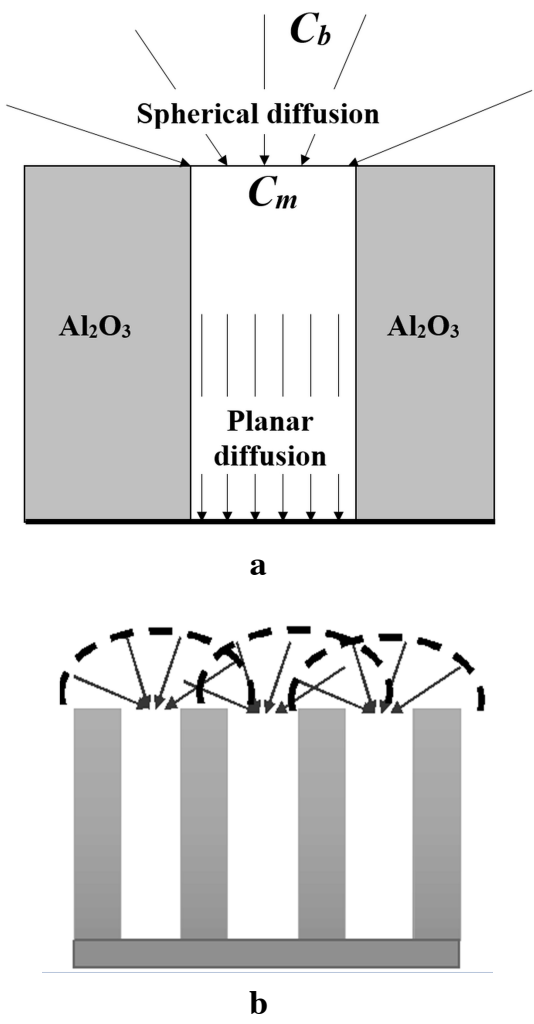

Fig. (4). Types of diffusion in the case of electrodeposition in the nano- and mesopores: (a) the two types of diffusion appear at time $t \geq t_{\text {tr }}$ (Eq. 5); (b) the overlapping spherical diffusion boundaries over mouths of pores at much longer times than $t_{\text {tr. }}$ a spherical diffusion for the longer time when the steady-state current density is achieved appears. 
However, diffusion-controlled reactions would not be the most desirable scenario to alloys electrodeposition into nanopores due to depletion of the solution and excessive side reactions. The transfer from mass-transport control to kinetic control of electrode processes is a powerful tool to electrodeposit nanostructures in the pores having a uniform length. It is achieved by proper selection of pulse deposition conditions [89]. In addition, pulse deposition mode is often used to improve the properties of electrodeposits such as morphology, crystallite size, chemical composition, crystalline structure and/or hydrogen inclusion [93, 94], and are widely used for the electrodeposition in the recesses [51, 60, 95]. By changing the frequency (or duty cycle), it is possible to provide either kinetic or diffusion (or mixed) control of electrode reaction. For frequency domain estimation, electrochemical impedance spectroscopy (EIS) data can be used. At high frequencies, the Warburg impedance is small since diffusing reactants do not have to move very far; at low frequencies the reactants have to diffuse farther, thereby increasing the Warburg (diffusion) impedance. The typical shape of the Nyquist diagram for Randles-Ershler impedance is shown in Fig. (5). At a critical frequency $\left(\omega_{\text {crit }}\right)$, the control over the electrochemical reaction switches from kinetic to diffusion and vice versa.

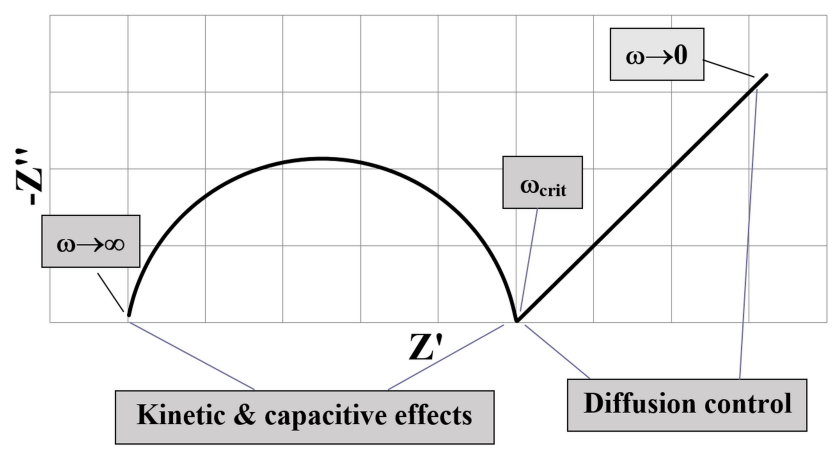

Fig. (5). Nyquist diagram for Randles circuit including linear diffusion.

However, in many cases, the kinetics of metals electrodeposition is rather complicated because of the adsorption on the electrode of intermediates and solution components (e.g., ligands), coupled with charge transfer and diffusion processes, and the diffusion impedance can be hidden from the obtained spectra [96]. Therefore, it is impossible to distinguish on the obtained EIS spectra the frequency ranges for which the electrode processes occur under kinetics or the mass transfer control. Simulation of EIS is a convenient way to estimate frequencies for which diffusion or kinetic limitations take place. It was shown [89], that simulation can be restricted to use a Randles-Ershler equivalent circuit containing the following elements: solution resistance, double layer capacitance, faradaic charge transfer resistance, and Warburg impedance. This impedance depends on the frequency of the potential perturbation and mass transfer conditions (concentrations, diffusion coefficients, and type of diffusion-semiinfinite or bounded diffusion). It was shown, that the values of $\omega_{\text {crit }}$ depend on the coefficient of diffusion and the charge transfer resistance, and in the most cases $\omega_{\text {crit }}>0.3-1.0 \mathrm{~Hz}$. This result means that if pulse current (or potential) cycle is
$1 \mathrm{sec}$ or shorter, the electrodeposition occurs under kinetic control, and it results in a more uniform length of nanowires (see Fig. 6). Uniformity of the nanowires, in this case, means the equal length of all wires inside the arrays.

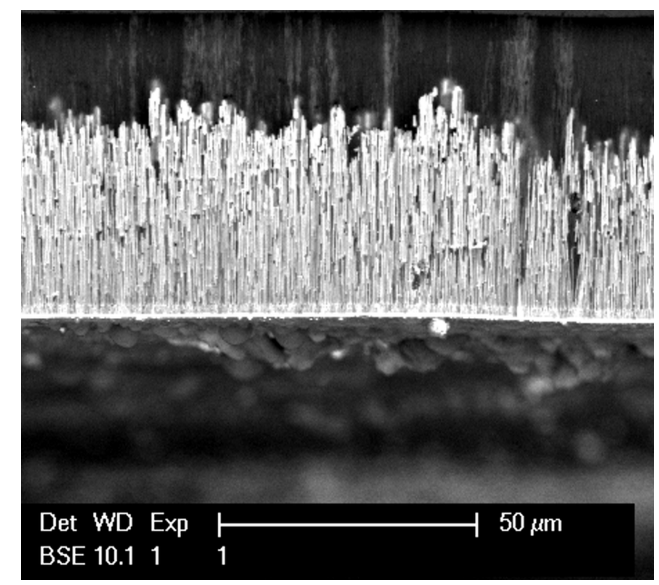

a

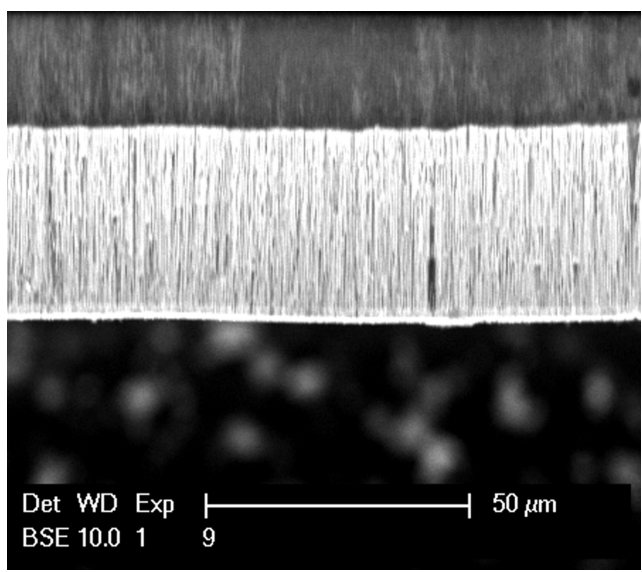

b

Fig. (6). SEM images of Co-W nanowires electrodeposited under: (a) galvanostatic and (b) current pulse (current ON $0.2 \mathrm{~s}$, OFF 0.8 s) modes.

\section{IRON GROUP ALLOY NANOWIRES PREPARED WITHIN AAO TEMPLATES}

Alloy electrodeposition in recessed substrates, such as in nanoporous membranes, not only needs to overcome the challenge of managing gas evolving side reactions but also there may be a challenge in controlling alloy composition, dictated by the individual reduction rates of each type of reacting metal ions. Under kinetic control, anomalous and induced codeposition behavior can occur. According to Brenner [97], if a 50:50 mixture of two metal ion components are mixed together in a non-complexing electrolyte, it is expected that the resulting composition of the deposit would be similar if the reaction is thermodynamically controlled. Under a purely kinetic control, the less noble component may deposit preferentially (i.e., anomalous codeposition) or one component may require the presence of another to deposit at all (i.e., induced codeposition). One way to assess the reduction behavior of metal ions is to ex- 
amine the partial current densities. A review of the partial current density behavior of alloy electrodeposition is best summarized by Landolt [98] that considers the interplay of kinetic limitations, and the influence of mass transport effects as well.

The reaction rate, or partial current density, of one metal ion species, can be inhibited, as in the case of anomalous codeposition, or it can be accelerated, as in the case of induced codeposition. Both of these behaviors have been shown to be $\mathrm{pH}$ dependent so that if there is varying local $\mathrm{pH}$ within a nanoporous template, this can affect the metal ion reduction rates and hence composition. For these reasons and due to changes in the resulting potential (at galvanostatic deposition), the electrolytes and deposition conditions applied for film alloy electrodeposition need to be adopted for nanowires electrodeposition.

Local $\mathrm{pH}$ changes can be minimized by using buffers and/or by implementing pulse deposition. For example, boric acid is commonly used in many electrolytes, in part, for this reason. Additives, such as sodium lauryl sulfate (SLS), affect the interfacial gas-liquid surface tension of bubbles that are generated from the side reaction, which then can indirectly change the local $\mathrm{pH}$ due to changes in the side reaction. While studies do not typically examine these variables per se many reported deposition electrolytes contain buffers and implement pulse deposition either in recognition of this issue or simply because it works.

\subsection{Binary nanowires}

\subsubsection{Fe-Ni nanowires}

The electrodeposition of alloys containing Ni-Fe is of interest due to their soft magnetic properties, such as those associated with (Permalloy, $\mathrm{Ni}_{80} \mathrm{Fe}_{20}$, or due to a low coefficient of thermal expansion (CTE), such as those exhibited in Invar alloys, $\mathrm{Fe}_{64} \mathrm{Ni}_{36}$ ). $\mathrm{Ni}-\mathrm{Fe}$ codeposition is often used as a model example of anomalous codeposition

Llavona et al. [99] electrodeposited Ni-Fe alloys into nanoporous templates and found an enhancement of anomalous deposition compared to the thin films in the potential range from -0.9 to $-1.2 \mathrm{~V} v s$. $\mathrm{Ag} / \mathrm{AgCl}$. In other words, there was more $\mathrm{Fe}$ in the deposited nanowires than in a thin film deposited using the same applied potential. However, when the applied potential was more negative, the composition of the nanowires was the same as the thin films. These results were explained by the interplay of kinetic control, where anomalous codeposition occurs, and diffusion. At low over potentials, there is more $\mathrm{Fe}$ on account of the anomalous deposition effect, but at high over potentials, iron and nickel ions are under diffusion control and their composition does not change with a further increase in over potential. Changes in the composition of the nanowires in the direction of growth were examined. The composition was uniform when the two metal reduction reactions were kinetically controlled, non-uniform when there was a mix of kinetic and diffusion control, and then uniform again when the nickel and iron ions were both deposited at their diffusion limiting current densities. The alloy composition varied in regions where hydrogen bubbles were lodged in the deposit. It was more Ni-rich near depressions in the deposit created by bubbles and the local change of composition was attributed to local current distribution effects. It was suggested that the compositional change was attributed to partial blocking of adsorbed species, a widely held view of the origin of anomalous codeposition [100-102].

Dragos et al. [103] also observed the anomalous codeposition behavior in the electrodeposition of $\mathrm{Fe}-\mathrm{Ni}$ nanowires into AAO templates with pulsed potential deposition from a sulfate-chloride electrolyte. Their electrolyte was composed of $0.4 \mathrm{M}$ boric acid, $0.3 \mathrm{M}$ ammonium chloride, $0.2 \mathrm{M}$ nickel sulfate, $0.02 \mathrm{M}$ iron sulfate, $5 \mathrm{mM}$ saccharin as a Na-salt, and $0.3 \mathrm{mM}$ sodium lauryl sulfate at a $\mathrm{pH}$ of 3 . As reported for $\mathrm{Ni}-\mathrm{Fe}$ codeposition in thin films, the Fe concentration in the deposits increased with the applied potential, reaching a maximum, and then decreasing. This relationship was similar to that reported in [99]. Dragos et al. [103] proposed that mass transport is only one of the factors influencing anomalous codeposition. Adsorption/desorption of iron and nickel intermediate electroactive species can be responsible for the nanowires composition. The adsorbed nickel species dissociates into solution preferentially while $\mathrm{FeOH}_{a d s}^{+}$accumulates on the surface. $\mathrm{Ni}_{56} \mathrm{Fe}_{44}$ nanowires with fcc structure and a grain size between 9-11 nm were formed.

Atalay et al. [104] reported on the electrodeposition of Ni-rich Ni-Fe alloys into highly ordered AAO templates, created by anodization, with pores sizes of $200 \mathrm{~nm}$. The sulfate electrolyte containing $7 \mathrm{mM}$ saccharine and $0.1 \mathrm{~g} / \mathrm{L}$ sodium lauryl sulfate at low $\mathrm{pH}$ was used for electrodeposition at an applied potential of $-2 \mathrm{~V} v s . \mathrm{Ag} / \mathrm{AgCl}$. The amount of nickel in the electrolyte was $0.1 \mathrm{M}$ while that of $\mathrm{Fe}$ was $0.005 \mathrm{M}$, a ratio of $\mathrm{Ni} / \mathrm{Fe}$ of 20 . The average alloy nanowire composition for all their deposits was between a ratio of 1 to 5 , an anomalous codeposition effect. The average deposit composition changed with deposition time, indicating a change of composition along the wire length. For example, at an electrolyte $\mathrm{pH}$ value of 2.0 and a deposition time of $7200 \mathrm{~s}$ the $\mathrm{Ni} / \mathrm{Fe}$ deposit ratio was $72: 28$, or 2.6 , while for a longer deposition time of $11000 \mathrm{~s}$ the deposit Ni/Fe ratio was nearly doubled. They attributed their observed anomalous composition as due to a hydroxide suppression mechanism, as first proposed by Dahms and Croll [105], where the local $\mathrm{pH}$ rise leads to the enhancement of Fe due to the precipitation of an iron hydroxide that inhibits Ni. In thin film deposition, this model has some limitations, as the degree of $\mathrm{pH}$ rise is typically not large enough to form the needed hydroxide blocking species. However, in a deep nanopore, the local $\mathrm{pH}$ rise is expected to be greater than near flat cathode.

\subsubsection{Co-Ni, Co-Fe nanowires}

Zhu et al. [106] electrodeposited Co-rich, Co-Ni alloys into an alumina template with a pore depth of approximately $\sim 50 \mu \mathrm{m}$, and a diameter of $200 \mathrm{~nm}$ using a sulfate-boric acid electrolyte. The magnetic properties of the obtained wires were affected by an applied magnetic field during the deposition. Vilana et al. [107] deposited Co-Ni nanowires into both polycarbonate and AAO nanoporous templates. The content of Co was shown to be somewhat higher in the case of polycarbonate templates. The wires were electrodeposited from a chloride- boric acid electrolyte. The structure was strongly influenced by the applied potential. Cobalt-rich hcp Co-Ni 
films were formed at more noble potentials and an fcc structure was observed at more negative deposition potentials. The magnetic properties were very different for two membranes. This is associated with the difference of the pore densities. In AAO the high pore density permits interaction of the magnetic moments of neighboring nanowires which increases the magnetic anisotropy, in contrast to nanowires fabricated in polycarbonate membranes.

Zhan et al. [108] prepared $\mathrm{Fe}_{1-\mathrm{x}} \mathrm{Co}_{\mathrm{x}}(0.0 \leq \mathrm{x} \leq 1.0)$ nanowires with an average size of $20 \mathrm{~nm}$ in diameter at different ratios of $\mathrm{Fe}^{2+/} \mathrm{Co}^{2+}$ species in the electrolyte. An $\mathrm{AC}$ (alternative current) current condition was used, $200 \mathrm{~Hz}$ and a cell potential of $15 \mathrm{~V}$ (AC). Elbaile et al. [109] reported pulse deposition to fabricate Fe-Co alloys. Compared to galvanostatic and potentiostatic deposition, pulse deposition was more beneficial to control the length and uniformity of nanowires. Khan and Petrikowski [110] electrodeposited Corich, Co-Fe alloys into anodized $\mathrm{Al}(1 \% \mathrm{Mg})$ nanoporous templates. They used a sulphate electrolyte and ac electrolysis (cell potential $20-25 \mathrm{~V} 50 \mathrm{~Hz}$ ). They were able to prepare thin nanowires, having diameters of $78 \mathrm{~nm}$ and $18 \mathrm{~nm}$. Arrays of nanowires show the perpendicular anisotropy accompanied with larger coercivity stemming from their reduced diameter when compared to the continuous films with similar compositions. Han et al. [111] reported the preparation of the Fe-Co nanowires under a magnetic field of $8 \mathrm{kOe}$, which induced a preferred crystallographic orientation, and affected the magnetic properties of the nanowires. Song et al. [112] electrodeposited thin Co-Fe nanowires into AAO templates of $50 \mathrm{~nm}$ diameter at different electrolyte temperatures; increasing the temperature resulted in a larger nanowire Co content. They used a sulfate electrolyte composed of $0.2 \mathrm{M}$ $\mathrm{CoSO}_{4}, 0.15 \mathrm{M} \mathrm{FeSO}_{4}, 0.4 \mathrm{M}$ boric acid and $0.05 \mathrm{~mol} / \mathrm{L}$ ascorbic acid. Co content increased from 52 at. \% to 77 at. $\%$ with the increase of the electrolyte temperature from $20^{\circ} \mathrm{C}$ to $60^{\circ} \mathrm{C}$. The lower electrolyte temperature contributed to the formation of single crystalline nanowires.

\subsubsection{Ternary Alloy Nanowires}

There are few reports of Fe-Ni-Co alloys [113-115]. A closely related system with a small amount of copper, Fe$\mathrm{Co}-\mathrm{Ni}-\mathrm{Cu}$, was fabricated by Huang et al. [113] by electrodeposition into commercial AAO templates. Pulse deposition was used to minimize the local $\mathrm{pH}$ rise. The obtained alloy was Co-rich. Fe-rich, Fe-Ni-Co nanowires were firstly reported by $\mathrm{Kim}$, et al. [70], from a sulfate/sulfamate electrolyte utilizing pulse deposition into commercial AAO templates. The target composition was close to bulk Super Invar, with the expectation of generating a nanowire with low thermal expansion. A five-segmented wire was electrodeposited, containing a sandwich with a Super Invar layer, separated by gold, followed by $\mathrm{Cu}$ and then another gold layer, Super Invar/Au/Cu/Au/Super Invar. In a narrow $\mathrm{pH}$ range in a citrate-boric acid solution, the copper layer can be selectively etched resulting in a gapped wire. Similarly, Geng and Podlaha [114] continued this work. Their new method included the formation of $\mathrm{Fe}-\mathrm{Ni}$-Co nanowire segments, followed by a copper etching. Li and Podlaha investigated the effect of hydrogen evolution reaction on the morphology and composition of DC electrodeposited Fe-Ni-Co wires or tubes formation by varying electrolyte $\mathrm{pH}$ and the concentration of sodium lauryl sulfate [115].

Samanifar et al. [116] electrodeposited Fe rich Fe-Co-Ni nanowires into AAO templates with a diameter of $175 \mathrm{~nm}$ at a constant current density of $-70 \mathrm{~mA} / \mathrm{cm}^{2}$. A reduction/oxidation voltage for $2.4 / 2.4 \mathrm{~ms}$ and off-time of $48 \mathrm{~ms}$ was applied to thin the barrier layer at the bottom of AAO templates. Although a slight increase of the Fe content was detected as the length of nanowires increased from 5 to 40 micron, the overall deposits had a relatively constant composition of $\mathrm{Fe}_{47} \mathrm{Co}_{38} \mathrm{Ni}_{15}$. Saedi and Ghorbani [117] demonstrated an approach to modify the barrier layer induced during the oxidizing process in order to obtain uniform aligned arrays. The composition of the resulting nanowires was found to be dependent on the thickness of AAO templates.

\section{PROPERTIES OF THE ARRAYS OF NANOS- TRUCTURES}

\subsection{Catalytic Properties of Nanowires}

The most frequent use in the view of catalytic prospects of the alloys of iron group metals is related to water splitting. Effective hydrogen production by water electrolysis is studied worldwide as an alternative to conventional sources of energy $[118,119]$. The research on effective electrodes for hydrogen evolution reaction (HER) is still in demand due to the more expensive cost of platinum-based electrodes. Electrocatalytic activity of nickel-based electrodes, including Ni$\mathrm{P}$ alloys towards water electrolysis, has been widely studied by several groups [120-122]. One of the factors influencing the catalytic activity is specific hydrogen adsorption, which depends on the electrode surface [123]. That depends on the geometry of the substrate used for electrodeposition. 1D geometry as nanowires or nanotubes is favorable in this respect. Even when having an identical chemical composition as films or coatings, nanowires/nanotubes can behave completely differently.

The replacement of $\mathrm{Ni}$ films by nanowires increases the electrocatalytic properties significantly [44]. The choice of appropriate nanowire material ( $\mathrm{NiP}$ instead of $\mathrm{Ni}$ ) and tuning nanowire length can considerably improve catalytic properties $[53,124])$. In order to enhance the catalytic activity of nanostructured electrodes towards HER magnetic field can be applied [125, 126]. Effect of externally applied magnetic field depends on the distance between electrodes, electrolyte concentration, which can be attributed to electrolyte convection caused by magneto-hydrodynamics. Thus, the influence of magnetic field on the electrocatalytic activity has been studied during cathodic polarization measurements for three different morphologies of the electrodes: plane, nanopattern, and nanowire [120]. Ni-P electrode has higher electrocatalytic activity than $\mathrm{Ni}$ electrode with corresponding electrode morphology, in the absence of an employed magnetic field. The different pictures are noticed when a magnetic field is applied. In this case, Ni-P alloys have a superior electrocatalytic activity in comparison to $\mathrm{Ni}$ electrodes which could be associated with the electron transfer in Ni-P $[15,127]$ and crystalline texture evolution with $\mathrm{P}$ content $[128,129]$ that give the enhancement of the catalytic property. 
However, the catalytic behavior changes for Ni nanoelectrodes under magnetic field due to the stronger coercivity, which induces higher magnetic field near the electrode and thus higher current densities. As a result, the Lorentz force facilitated the outflow of hydrogen bubbles from the cathode surface and promotes the HER reaction. Furthermore, for both $\mathrm{Ni}$ and Ni-P electrodes, the electrocatalytic activity evidently increased in the following sequence of the electrodes: plane, nanopattern, and nanowire. These outcomes are directly connected to the higher specific surface area of the nanostructured electrodes $[120,130]$. However, nanopatterned electrode exhibited the higher catalytic activity than plane electrode in spite of the lower specific area. It seems that, near the perimeter of the nanopattern, the electric field concentration can change the overall current efficiency, leading to the increase of HER activity [53, 131]. The bubbles generated from the nanopattern electrode were smaller and more uniform in size distribution [120]. The smaller bubbles escape faster from the nanopatterned electrode. In the case of the plane electrode, the larger bubble takes more time to grow and blocks the electrode surface decreasing its catalytic activity.

Not only phosphide-based nanomaterials could be used as effective electrodes; sulfides and even selenides of iron group metals also manifest promising behavior. These catalysts are cheaper than the oxides and hydroxides of the same type $[119,132,133]$.

The multilayered structures containing iron group metals also can catalyze electro-oxidation of methanol. The $\mathrm{Pt} / \mathrm{Ni}$ multilayer nanowire electrode was obtained which demonstrated better methanol electrooxidation catalytic activity than the pure Pt nanowires: the peak current density for $\mathrm{Pt} / \mathrm{Ni}$ nanowires reached $0.258 \mathrm{~mA} / \mathrm{cm}^{2}$, which is higher than that for pure $\mathrm{Pt}\left(0.102 \mathrm{~mA} / \mathrm{cm}^{2}\right)$. Moreover, these multilayered nanowires were more stable to poisoning by CO-like intermediates from dehydrogenation of methanol than Pt. This is important since one of the drawbacks of Pt electrodes is, indeed, the fast poisoning by $\mathrm{CO}$, which reduces substantially the effective use of such electrodes [134].

Similar results were obtained also for PtCo nanowires [135] which showed better performance for methanol electro-oxidation than PtCo films with the same composition. Of course, this effect is due, in part, to the higher specific area of nanowires compared to films, but the authors also indicated that the nanostructured configuration of $\mathrm{PtCo}$ results in better resistance to $\mathrm{CO}$ poisoning than in films. The last outcome is not well understood yet but seems to be linked to the hypothesis that $\mathrm{CO}$ is oxidized easier on larger Pt particles than on smaller ones [129]. Here, we can underline that in both cases, PtNi and PtCo nanowires, were successfully obtained using AAO template deposition. Moreover, $\mathrm{Co}-\mathrm{W}$ and $\mathrm{Fe}-\mathrm{W}$ nanowires could have also a high catalytic activity for methanol electro-oxidation as their plane electrodes have better performance than Pt electrodes [136].

\subsection{Magnetic Properties of Nanowires}

The magnetic properties of arrays of nanowires deposited inside the pores of oxide templates $\left(\mathrm{AAO}\right.$ or $\left.\mathrm{TiO}_{2}\right)$ have been widely investigated for applications like high-density recording media [48], giant magneto-resistance [137] or magnetic control of biomolecule desorption [138], among others. Eventually, polymeric porous templates (e.g., nanoporous polycarbonate membranes) have been utilized, in cases where the use of oxide membranes was found to be not compatible with the electrolytic bath and deposition conditions $[139,140]$. The investigated magnetic materials comprise single metals (like Fe, Co or Ni) [11, 48, 51, 141-144], binary (Co-W, Co-Pt, CoNi, FeNi, etc.) [51, 89, 106, 144154] and ternary alloys (e.g., CoNiP) [155].

Core-shell structures [156] and multi-segmented magnetic nanowires [137-140, 157-159] have been also investigated. Introducing nanoporosity into the architecture of the magnetic nanowires offers the potential to take advantage of magnetism in applications like catalysis or electrocatalysis [158, 160].

In general, the properties of magnetic nanowires differ from the bulk magnetic behavior of materials with the same composition owing to: (i) effects stemming from the reduced lateral dimensions of the nanowires (sample size effects), (ii) influence of interwire dipolar interactions between the nanowires, when they are still inside the templates (which cause noise in applications like recording media) [161] or once released into a suspension (which causes aggregation of the nanowires) [138].

An insulated magnetic nanowire usually exhibits a larger coercivity than a bulk material with the same composition ("sample size effect"). This is due to a reduction in the number of domain walls inside nanowires (particularly if they are sub-50 $\mathrm{nm}$ in diameter) and the predominance of singledomain magnetization reversal (coherent rotation). Other types of magnetic configurations are sometimes formed in the nanowires, like magnetic vortices or even more complex arrangements, particularly for diameters exceeding $200 \mathrm{~nm}$ [162].

In any case, the high-aspect ratio of magnetic nanowires usually causes the occurrence of shape anisotropy, making them promising candidates for recording media with perpendicular-to-plane magnetic anisotropy [48, 137]. In some cases, particularly for single crystalline nanowires (e.g., Co nanowires), the magnetocrystalline axis of easy magnetization is not oriented along the nanowire axis but perpendicular to it $[51,143,163,164]$. The competition between shape anisotropy and magnetocrystalline anisotropy causes peculiar angular dependence of the magnetic response in this case.

Dipolar interactions are particularly pronounced for highly-packed arrays of nanowires, both for ordered and disordered arrangements. Dipolar interactions depend on both the interwire lateral spacing (pitch size) and the length of the nanowires (see Fig. 7). The net effects of interwire dipolar interactions in nanowires embedded in a template are to decrease the coercivity and the remanence and to induce changes in the slope of the hysteresis loops [163, 164].

More specifically, for an array of nanowires (still embedded in the AAO template) the following three contributions will have an effect on the easy axis direction in the measured hysteresis loops: (i) the shape anisotropy of the 

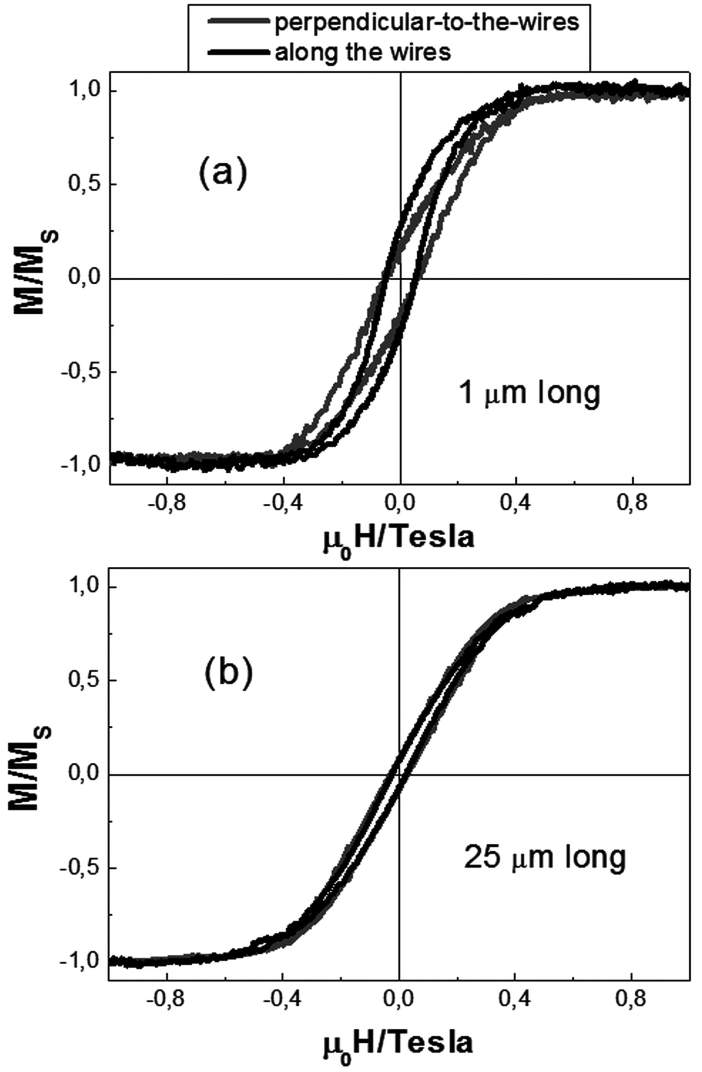

Fig. (7). Hysteresis loops, measured along and perpendicular to the nanowires axis, corresponding to arrays of $\mathrm{Co}-\mathrm{W}$ nanowires with two different nanowire length, (a) $1 \mu \mathrm{m}$ and (b) $25 \mu \mathrm{m}$ [89]. The decrease of coercivity, remanence, and slope of the hysteresis loops observed for the longer nanowires is due to stronger interwire magnetic dipolar interactions.

nanowires (which depends on their length-to-diameter aspect ratio), (ii) the magnetocrystalline anisotropy (whose direction depends on crystallographic structure and texture) and (iii) the magnetostatic coupling among neighbouring nanowires (which depends on the interwire dipolar interactions and promotes a magnetic easy axis perpendicular to the nanowire axis).

To take all these effects into account, one can assume a two-dimensional infinite array of cylindrical particles with radius $r$, length $L$, aspect ratio $A_{r}=L / 2 r$ and interwire distance $D$. One can then consider that the total dipolar field generated when all nanowires are oriented along the nanowire axis direction can be written as $[146,147,164$, 165]:

$$
H_{d i p, 0^{\circ}}=\frac{4.2 M_{S} \pi r^{2} L}{D^{3}}
$$

On the contrary, if all moments are aligned perpendicular to the direction of the wire, then the created dipolar field would be:

$$
H_{d i p, 90^{\circ}}=\frac{-2.1 M_{S} \pi r^{2} L}{D^{3}}
$$

The self-demagnetizing magnetic field of one nanowire can be then expressed as:

$$
H_{\text {demag }}=\left(N_{\perp}-N_{\|}\right) M_{S}
$$

Where, $N_{\|}$and $N_{\perp}$ are the demagnetizing factors along the nanowire axis and its perpendicular direction and can be calculated using a prolate spheroid approximation, as follows [144]:

$$
\begin{aligned}
& N_{\|}=\frac{4 \pi}{A_{r}^{2}-1}\left[\frac{A_{r}}{2 \sqrt{\left(A_{r}^{2}-1\right)}} \ln \left(A_{r}+\sqrt{A_{r}^{2}-1}\right)-1\right] \\
& N_{\perp}=\frac{4 \pi-N_{\|}}{2}
\end{aligned}
$$

Hence, the total effective anisotropy field can be then expressed as:

$$
H_{K, e f f}=\left(N_{\perp}-N_{\|}\right) M_{S}-\frac{6.3 M_{S} \pi r^{2} L}{D^{3}}+H_{m c}
$$

This equation predicts that if $H_{K, \text { eff }}$ is positive the magnetic easy axis will be parallel to the wires axis; conversely, for negative $H_{K, \text { eff }}$, the magnetic easy axis will tend to be oriented along the perpendicular-to-nanowires axis. $H_{m c}$ represents the magnetocrystalline anisotropy contribution, which is usually small compared to the other terms of the equation. If one uses approximate values of the nanowire length and diameter and the distance between nanowires (pitch size), one can try to estimate the effective anisotropy field of a given array of nanowires using Eq. (8). Hence, this simple model is capable of describing, in a semi-quantitative manner, the preferred orientation of the magnetic easy axis in the hysteresis loops of an array of magnetic nanowires.

Remarkably, in nanowires comprising more than one material with dissimilar magnetic properties (e.g., core-shell nanowires), effects like exchange bias (i.e., a shift of the hysteresis loop along the magnetic field axis) are sometimes observed, due to the coupling between the two magnetic components (e.g., exchange interactions between ferromagnetic Co core with an antiferromagnetic $\mathrm{CoO}$ shell) [156].

Multisegmented nanowires comprising alternating ferromagnetic and non-magnetic segments (e.g., $\mathrm{Fe} / \mathrm{Cu}$ multilayered nanowires) can be used as magnetic sensors, taking advantage of the giant magnetoresistance effect. The resistivity of these nanowires depends on whether the ferromagnetic segments are oriented parallel or antiparallel to each other [137]. Hence, it is clear that the functionalities of magnetic nanowires can be enhanced by combining different materials in the nanowire architecture. In this sense, it might be also possible to combine magnetic counterparts with materials exhibiting other interesting properties (i.e., photocatalytic or photoluminescent segments). In these cases, the magnetic constituents can be used to magnetically guide (wirelessly, with external magnetic fields) the nanowires to specific locations where the catalytic reactions (or other applications like drug delivery or biological labelling) could take place. In any 

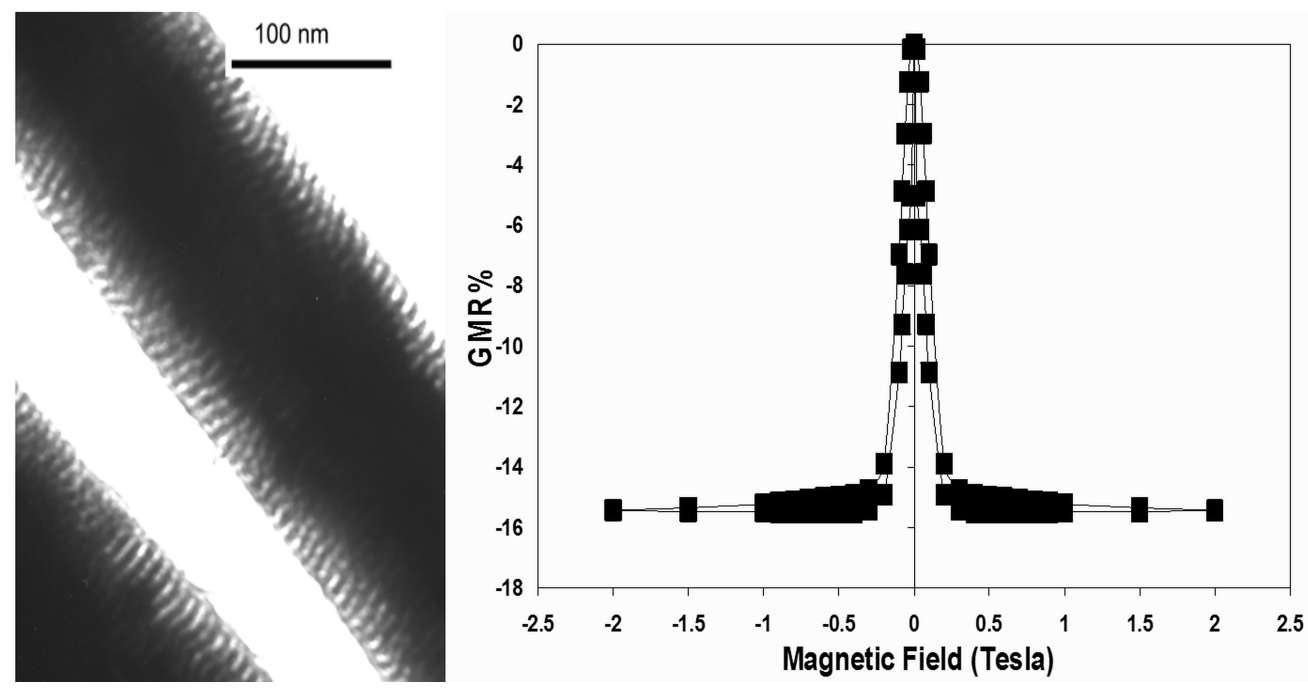

Fig. (8). Co-rich, $\mathrm{Fe}-\mathrm{Co}-\mathrm{Ni}-\mathrm{Cu} / \mathrm{Cu}$ multilayered nanowire and its accompanying GMR from an array of nanowires in AAO with permission from The Electrochemical Society [171].

case, however, for the full exploitation of the technological potential of magnetic nanowires (single component or multicomponent), it is necessary to combine deep knowledge on the magnetic behavior of this type of materials together with precise control of the synthetic procedures to obtain the desired nanoarchitectures.

In addition, the unique combination of a magnetostrictive effect and high magnetic permeability offers potential applications of the nanowires kept in templates as ultrasonic transducers and sonar sensors [166], perpendicular magnetic storage devices $[46,167]$.

\subsection{Multilayered Nanowires}

The 2007 Nobel Prize in physics was awarded to Fert and Grünberg, for their discovery and fundamental understanding of giant magnetoresistance (GMR). Although this was not first demonstrated with electrodeposited materials, it was later shown that electrodeposition could be a viable process and perhaps a niche in developing GMR nanowires using the template approach. To create a GMR effect a ferromagnetic layer, with nanoscale thickness, is sandwiched between a non-ferromagnetic (paramagnetic) layers. A comprehensive review of electrodeposited, GMR thin films is provided by Bakonyi and Peter [168]. Electrodeposition is uniquely suited to fabricate these layered structures in the form of nanowires. While Alper et al. [169] were the first to demonstrate an electrodeposited GMR film, it was Blondel et al. [43] that demonstrated a significant GMR property in nanowires fabricated with electrodeposited sequential layers of $\mathrm{Co}$ and $\mathrm{Cu}$ layers, and $\mathrm{Ni}-\mathrm{Fe}$ and $\mathrm{Cu}$ layers, modulated to create a series of multilayers $(\mathrm{Co} / \mathrm{Cu}$ and $\mathrm{Ni}-\mathrm{Fe} / \mathrm{Cu})$. They used a single, sulfate-boric acid electrolyte to deposit the two different layers with a modulation of the applied working electrode potential. A more noble potential was used to deposit the $\mathrm{Cu}$ layer and the less noble to deposit the magnetic layer (Co or $\mathrm{Ni}-\mathrm{Fe})$. Although this seminal work was done using polycarbonate track-etched templates, other researchers have shown that the procedure can also be readily adapted to AAO membrane templates. For example, Evans et al. [170] electrodeposited $\mathrm{Co}-\mathrm{Ni}-\mathrm{Cu} / \mathrm{Cu}$ nanowires within commercially available AAO templates with a pore size of $20 \mathrm{~nm}$. The selected deposition potential of the less noble, ternary alloy layer was deposited at an applied potential of $1.6 \mathrm{~V} v s$. SCE, whereas a pure $\mathrm{Cu}$ layer was deposited at -0.2 $\mathrm{V}$ vs. SCE. An annealing process was used to modify and improve the GMR property.

Electrodeposition of $\mathrm{FeCoNiCu} / \mathrm{Cu}$ nanometric, compositionally modulated alloy nanowires was reported by Huang et al. [113]. Pure $\mathrm{Cu}$ nanowires and $\mathrm{Co}$-rich $\mathrm{FeCoNiCu}$ alloy nanowires were deposited from a single electrolyte. A double potential pulse scheme was used for the multilayer deposition and significant anodic dissolution was observed during the low potential pulse. Galvanostatic triple pulses with a relaxation period were developed to minimize the local $\mathrm{pH}$ rise inside the nanopores during fabrication of the layered $\mathrm{FeCoNiCu} / \mathrm{Cu}$ nanowires. Fig. (8) shows a layered nanowire region containing $\mathrm{Co}$-rich $\mathrm{CoNiFeCu} / \mathrm{Cu}$ layers fabricated within $60 \mu \mathrm{m}$ thick, commercially available anodized aluminum oxide membranes deposited from a tartrate sulfamic acid electrolyte. Fig. (8), clearly shows the layering effect necessary for GMR. The TEM image corresponds to a GMR behavior as depicted in Fig. (8). The light colored layer is $\mathrm{Cu}$, having a thickness of $4.2+0.7 \mathrm{~nm}$ and the dark layer is the $\mathrm{CoNiFeCu}$ alloy of thickness $5.6+0.4 \mathrm{~nm}$. In addition to $\mathrm{FeCoNiCu} / \mathrm{Cu}$ nanowires, nanotubes were also prepared at a low electrolyte temperature from a relatively dilute electrolyte [171].

\section{CONCLUSION}

Progress on the template electrodeposition of iron-group alloys nanowires into oxide membranes is reviewed. Three aspects that play a role during the growth of this type of materials were analyzed in detail: barrier layer challenges during electrodeposition into on-substrate anodic alumina/Al templates; hydrogen evolution from the nanopores, and mass transport inside the pores and its critical importance to obtain uniform nanostructures. Works dealing with electrodeposi- 
tion of binary and ternary iron-group alloys confirm that controlling alloy composition inside nanopores is a challenge since one should control the individual reduction rates of each type of reacting metal ions. Previous works on catalytic hydrogen evolution and methanol oxidation on the nanowires arrays are also reviewed. From the point of view of magnetic properties, precise control of the composition and crystallographic structure of the nanowires, as well as their aspect ratio and interwire distance is crucial in order to obtain materials for a variety of applications, including fields like magnetic recording, GMR sensors or even biomedicine.

\section{ACKNOWLEDGEMENTS}

Authors acknowledge funding from $\mathrm{H} 2020$ projects (SMARTELECTRODES 778357 and SELECTA 642642). Also, partial support by Roche Diagnostics, the Spanish Government [Project MAT2017-86357-C3-1-R and associated FEDER], the Generalitat de Catalunya (2017-SGR$292)$, and the Moldavian national project (15.817.02.05A) is acknowledged.

\section{REFERENCES}

[1] Martin, C.R. Nanomaterials: A membrane-based synthetic approach. Science, 1994, 266(5193), 1961-1966.

[2] Penner, R.M.; Martin, C.R. Preparation and electrochemical characterization of ultramicroelectrode ensembles. Anal. Chem., 1987, 59(21), 2625-2630.

[3] Iijima, S. Helical microtubules of graphitic carbon. Nature, 1991, 354(6348), 56-58.

[4] Chopra, N.G.; Luyken, R.J.; Cherrey, K.; Crespi, V.H.; Cohen, M.L.; Louie, S.G.; Zettl, A. Boron nitride nanotubes. Science, 1995, 269(5226), 966-967.

[5] Dai, H.; Wong, E.W.; Lu, Y.Z.; Fan, S.; Lieber, C.M. Synthesis and characterization of carbide nanorods. Nature, 1995, 375(6534), 769-772

[6] Wang, Y.; Zhang, L.; Meng, G.; Liang, C.; Wang, G.; Suna, S. Zn nanobelts: A new quasi one-dimensional metal nanostructure. Chem. Commun., 2001, (24), 2632-2633.

[7] Vernickaite, E.; Bubniene, U.; Cesiulis, H.; Ramanavicius, A.; Podlaha, E.J. A hybrid approach to fabricated nanowirenanoparticle composites of a Co-W alloy and Au nanoparticles. $J$. Electrochem. Soc., 2016, 163(7), D1-D5.

[8] Possin, G.E. A method for forming very small diameters wires. Rev. Sci. Instrum., 1970, 41(5) 772-774.

[9] Kline, T.R.; Tian, M.; Wang, J.; Sen, A.; Chan, M.W.H.; Mallouk, T.E. Template-grown metal nanowires. Inorg. Chem., 2006, 45(19), 7555-7565.

[10] Hurst, S.J.; Payne, E.K.; Qin, L.; Mirkin, C.A. Multisegmented one-dimensional nanorods prepared by hard-template synthetic methods. Angew. Chem. Int. Ed., 2006, 45(17), 2672-2692.

[11] Prida, V.M.; Hernandez-Velez, M.; Cervera, M.; Pirota, K.; Sanz, R.; Navas, D.; Asenjo, A.; Aranda, P.; Ruiz-Hitzky, E.; Batallan, F.; Vazquezb, M.; Hernando, B.; Menendez, A.; Bordel, N.; Pereiro, R. Magnetic behaviour of arrays of Ni nanowires by electrodeposition into self-aligned titania nanotubes. J. Magn. Magn. Mater., 2005, 294(2), e69-e72
[12] Schonenberger, C.; van der Zande, B.M.I.; Fokkink, L.G.J.; Henny, M.; Schmid, C.; Kruger, M.; Bachtold, A.; Huber, R.; Birk, H.; Staufer, U. Template synthesis of nanowires in porous polycarbonate membranes: Electrochemistry and morphology. J. Phys. Chem. $B, 1997,101(28), 5497-5505$.

[13] Gerngross, M.-D.; Chemnitz, S.; Wagner, B.; Carstensen, J.; Föll, H. Ultra-high aspect ratio $\mathrm{Ni}$ nanowires in single-crystalline InP membranes as multiferroic composite. Phys. Status Solidi RRL, 2013, 7(5), 352-354.

[14] Gerngross, M.-D.; Carstensen, J.; Föll, H. Electrochemical growth of Co nanowires in ultra-high aspect ratio InP membranes: FFTimpedance spectroscopy of the growth process and magnetic properties. Nanoscale Res. Lett., 2014, 9(1), 316-325.

[15] Chu, S.-Z.; Wada, K.; Inoue, S.; Isogai, M.; Yasumori, A. Fabrication of ideally ordered nanoporous alumina films and integrated nanotubule arrays by high -field anodization. Adv. Mater., 2005, 17(17), 2115-2119.

[16] Stępniowski, W.J.; Salerno, M. Fabrication of nanowires and nanotubes by anodic alumina template-assisted electrodeposition, In: Manufacturing Nanostructures, Ahmed, W.; Ali, N.; Eds.: One Central Press, 2014, pp. 321-357.

[17] Tsyntsaru, N. Porous anodized aluminium oxide: Application outlooks. Chemija, 2016, 27(1), 17-23.

[18] Sulka, G.D.; Zaraska, L.; Stepniowski, W.J.; In: Encyclopedia of Nanoscience and Nanotechnology, $2^{\text {nd }}$ Ed., American Scientific Publishers, 2011, Vol. 11, pp. 261-349.

[19] Asoh, H.; Ono, S. Fabrication of ordered anodic nanoporous alumina layers and their application to nanotechnology. In: Electrocrystallization in Nanotechnology. Georgi Staikov Ed.: WILEYVCH Verlag GmbH \& Co. KGaA, Weinheim, 2007, pp. 138-165.

[20] Davydov, A.D.; Volgin, V.M. Template electrodeposition of metals. Review. Russ. J. Electrochem., 2016, 52(9), 806-831.

[21] Tsyntsaru, N.; Kavas, B.; Sort, J.; Urgen, M.; Celis, J.-P. Mechanical and frictional behaviour of nano-porous anodised aluminium. Mater. Chem. Phys., 2014, 148(3), 887-895.

[22] Thompson, G.E. Porous anodic alumina: Fabrication, characterization and applications. Thin Solid Films, 1997, 297(1-2), 192-201.

[23] Buijnsters, J.G.; Zhong, R.; Tsyntsaru, N.; Celis, J.-P. Surface wettability of macroporous anodized aluminum oxide. ACS Appl. Mater. Interfaces, 2013, 5(8), 3224-3233.

[24] Hu, J.; Zhang, F.; Wang, J.; Xiao, J.Q. Synthesis of singlecrystalline $\mathrm{Fe}$ nanowires using catalyst-assisted chemical vapor deposition. Mater. Lett., 2015, 160, 529-532.

[25] Sadki, E.S.; Ooi, S.; Hirata, K. Focused-ion-beam-induced deposition of superconducting nanowires. Appl. Phys. Lett., 2004, 85(25), 6206-6208.

[26] Zettler, J.K.; Hauswald, C.; Corfdir, P.; Musolino, M.; Geelhaar, L.; Riechert, H.; Brandt, O.; Fernández-Garrido, S. Hightemperature growth of $\mathrm{GaN}$ nanowires by molecular beam epitaxy: toward the material quality of bulk GaN. Cryst. Growth Des., 2015 , 15(8), 4104-4109.

[27] Vázquez, M. Ed. Magnetic nano- and microwires- design, synthesis, properties and applications. Cambridge, UK: Woodhead Publishing is an imprint of Elsevier, 2015.

[28] Ricelli, L.A.; Bozzini, B.; Mele, C.; D'Uzo, L. A review of nanostructural aspects of metal electrodeposition. Int. J. Electrochem. Sci., 2008, 3(4), 356-408.

[29] Erb, U. Electrodeposited vs. consolidated nanocrystals: Differences and similarities. Nanostruct. Mater., 1997, 9, 261-270.

[30] McMahon, G.; Erb, U. Structural transitions in electroplated Ni-P alloys. J. Mater. Sci. Lett., 1989, 8(7), 865-868.

[31] Grabchikov, S.S.; Potuzhnaya, O.I.; Sosnovskaya, L.B.; Sheleg, M.U. Microstructure of amorphous electrodeposited Co-Ni-W films. Russ. Metall-Metall-U, 2009, 2009(2), 164-171.

[32] Alfantazi, A.M.; Erb, U. Synthesis of nanocrystalline Zn-Ni alloy coatings. J. Mater. Sci. Lett., 1996, 15(15), 1361-1363.

[33] Ohgai, T.; Tanaka, Y.; Washio, R. Nanocrystalline structure and soft magnetic properties of nickel-molybdenum alloy thin films electrodeposited from acidic and alkaline aqueous solutions. $J$. Solid State Electr., 2013, 17(3), 743-750. 
[34] Doi, S.; Wang, F.; Hosoiri, K.; Watanabe, T. Preparation and characterization of electrodeposited Fe-Pd binary alloy film. Mater. Trans., 2003, 44(4), 649-652.

[35] Juskenas, R.; Valsiunas, I.; Pakstas, V.; Selskis, A.; Jasulaitiene, V.; Karpaviciene, V.; Kapocius, V. XRD, XPS and AFM studies of the unknown phase formed on the surface during electrodeposition of Ni-W alloy. Appl. Surf. Sci., 2006, 253(3), 1435-1442.

[36] Sun, L.; Hao, Y.; Chien, C.-L.; Searson, P.C. Tuning the properties of magnetic nanowires. IBM J. Res. Dev., 2005, 49(1), 79-102.

[37] Ramazani, A.; Asgari, V.; Montazer, A.H.; Kashi, M.A. Tuning magnetic fingerprints of FeNi nanowire arrays by varying length and diameter. Curr. Appl. Phys., 2015 15(7), 819-828.

[38] Samanifar, S.; Alikhani, M.; Almasi Kashi, M.; Ramazani, A.; Montazer, A.H. Magnetic alloy nanowire arrays with different lengths: Insights into the crossover angle of magnetization reversal process. J. Magn. Magn. Mater., 2017, 430, 6-15.

[39] Méndez, M; González, S.; Vega, V.; Teixeira, J.M.; Hernando, B.; Luna, C.; Prida, V.M. Ni-Co alloy and multisegmented Ni/Co nanowire arrays modulated in composition: Structural characterization and magnetic properties. Crystals, 2017, 7(3), 66.

[40] Zeeshan, M.A.; Pané, S.; Youn, S.K.; Pellicer, E.; Schuerle, S.; Sort, J.; Fusco, S.; Lindo, A.M.; Park, H.G.; Nelson, B.J. Graphite coating of iron nanowires for nanorobotic applications: Synthesis, characterization and magnetic wireless manipulation. Adv. Funct. Mater., 2013, 23(7), 823-831.

[41] Zhang, D.; Liu, Z.; Han, S.; Li, C.; Lei, B.; Stewart, M.P.; Tour, J.M.; Zhou, C. Magnetite $\left(\mathrm{Fe}_{3} \mathrm{O}_{4}\right)$ core-shell nanowires: Synthesis and magnetoresistance. Nano Lett., 2004, 4(11), 2151-2155.

[42] Piraux, L.; George, J.M.; Despres, J.F.; Leroy, C.; Ferain, E.; Legras, R.; Ounadjela, K.; Fert, A. Giant magnetoresistance in magnetic multilayered nanowires. Appl. Phys. Lett., 1994, 65(19), 2484-2486.

[43] Blondel, A.; Meier, J.P.; Doudin, B.; Ansermet, J.P. Giant magnetoresistance of nanowires of multilayers. Appl. Phys. Lett., 1994; 65(23), 3019-3021.

[44] Lee, J.K.; Yi, Y.; Lee, H. J.; Uhm, S.; Lee, J. Electrocatalytic activity of Ni nanowires prepared by galvanic electrodeposition for hydrogen evolution reaction. Catal. Today, 2009, 146(1-2), 188-191.

[45] Pirota, K.R.; Béron, F.; Zanchet, D.; Rocha, T.C.R.; Navas, D.; Torrejón, J.; Vazquez, M.; Knobel, M. Magnetic and structural properties of fcc/hcp bi-crystalline multilayer Co nanowire arrays prepared by controlled electroplating. J. Appl. Phys., 2011, 109(8), 083919.

[46] Kartopu, G.; Yalçın, O.; Es-Souni, M.; Başaran, A.C. Magnetization behavior of ordered and high-density Co nanowire arrays with varying aspect ratio. J. Appl. Phys., 2008, 103(9), 093915.

[47] Whitney, T.M.; Jiang, J.S.; Searson, P.C.; Chien, C.L. Fabrication and magnetic properties of arrays of metallic nanowires. Science, 1993, 261(5126), 1316-1319.

[48] Irshad, M.I.; Ahmad, F.; Mohamed, N.M. A review on nanowires as an alternative high density magnetic storage media. AIP Conf. Proc., 2012, 1482(1), 625-632.

[49] Xu, C.-L.; Li, H.; Zhao, G.-Y.; Li, H.-L. Electrodeposition of ferromagnetic nanowire arrays on $\mathrm{AAO} / \mathrm{Ti} / \mathrm{Si}$ substrate for ultrahighdensity magnetic storage devices. Mater. Lett., 2006, 60(19), 23352338.

[50] Valentini, F.; Palleshi, G. Nanomaterials and analytical chemistry. Anal. Lett., 2008, 41(4), 479-520.

[51] Schlorb, H.; Haehnel, V.; Khatri, M.S.; Srivastav, A.; Kumar, A.; Schultz, L.; Fahler, S. Magnetic nanowires by electrodeposition within templates. Phys. Status Solidi B, 2010, 247(10), 2364-2379.

[52] Wang, Y.; Liu, D.; Liu, Z; Xie, C.; Huo, J.; Wang, S. Porous cobalt-iron nitride nanowires as excellent bifunctional electrocatalysts for overall water splitting. Chem. Commun., 2016, 52(85), 12614-12617.

[53] Hsu, C.S.; Lee, H.B.; Lin, C.S.; Lee, C.Y. Study on the electrodeposition of Ni-P nanowires and their electrocatalytic properties. Metall. Mater. Trans. A, 2010, 41(3), 768-774.

[54] Mátéfi-Tempfli, S.; Mátéfi-Tempfli, M.; Piraux, L. Fabrication of nanowires and nanostructures: combining template synthesis with patterning methods. App. Phys. A, 2009, 96(3), 603-608.
[55] Reddy, S.M.; Park, J.J.; Na, S.-M.; Maqableh, M.M.; Flatau, A.B.; Stadler, B.J.H. Electrochemical synthesis of magnetostrictive $\mathrm{Fe}-$ $\mathrm{Ga} / \mathrm{Cu}$ multilayered nanowire arrays with tailored magnetic response. Adv. Funct. Mater., 2011, $21(24), 4677-4683$.

[56] Sharma, G.; Pishko, M.V.; Grimes, C.A. Fabrication of metallic nanowire arrays by electrodeposition into nanoporous alumina membranes: Effect of barrier layer. J. Mater. Sci., 2007, 42(13), 4738-4744.

[57] Furneaux, R.C.; Rigby, W.R.; Davidson, A.P. The formation of controlled-porosity membranes from anodically oxidized aluminium. Nature, 1989, 337(6203), 147-149.

[58] Santos, A.; Vojkuvka, L.; Pallarés, J.; Ferré-Borrull, J.; Marsal, L.F. In situ electrochemical dissolution of the oxide barrier layer of porous anodic alumina fabricated by hard anodization. J. Electroanal. Chem., 2009, 632(1-2), 139-142.

[59] Jagminas, A.; Kurtinaitienè, M.; Angelucci, R.; Valinčius, G. Modification of alumina barrier-layer through re-anodization in an oxalic acid solution with fluoride additives. Appl. Surf. Sci., 2006, 252(6), 2360-2367.

[60] Zhao, X.; Seo, S.K.; Lee, U.J.; Lee, K.H. Controlled electrochemical dissolution of anodic aluminium oxide for preparation of openthrough pore structures. J. Electrochem. Soc., 2007, 154(10), C553C557.

[61] Park, S.H.; Kim, S.; Lee, D.J.; Yun, S.; Khim, Z.G. Selective wetchemical etching of the barrier layer during formation of porous anodic aluminum oxide template. J. Electrochem. Soc., 2009, 156(11), K181-K185.

[62] Tsyntsaru, N. In: IFMBE Proceedings of the $3^{\text {rd }}$ International Conference on Nanotechnologies and Biomedical Engineering, Chisinau, Republic of Moldova, September 23-26, 2015; Sontea, V.; Tiginyanu, I., Eds. Springer-Verlag: Singapore, 2016 vol. 55, pp.123-126.

[63] Akinci, Z.B.; Urgen, M. A simple method for the production of aao templates for DC electrodeposition of nanostructures. ECS Electrochem. Lett., 2014, 3(10), D46-D49.

[64] Ali, G.; Maqbool, M. Fabrication of cobalt-nickel binary nanowires in a highly ordered alumina template via AC electrodeposition. Nanoscale Res. Lett., 2013, 8(1), 352.

[65] Hoare, J.P. On the role of boric acid in the Watts bath. J. Electrochem. Soc., 1986, 133(12), 2491-2494.

[66] Cesiulis, H.; Podlaha-Murphy, E.J. Electrolyte considerations of electrodeposited $\mathrm{Ni}-\mathrm{W}$ alloys for microdevice fabrication. Mater. Sci. Medzg., 2003, 9(4), 324-327.

[67] Donten, M.; Stojek, Z.; Osteryoung, J.G. Voltammetric, optical, and spectroscopic examination of anodically forced passivation of cobalt-tungsten amorphous alloys. J. Electrochem. Soc., 1993, 140(12), 3417-3424.

[68] Cesiulis, H.; Baltutiene, A.; Donten, M.; Donten, M.L.; Stojek, Z Increase in rate of electrodeposition and in $\mathrm{Ni}(\mathrm{II})$ concentration in the bath as a way to control grain size of amorphous / nanocrystalline Ni-W alloys. J. Solid State Electrochem., 2002, 6(4), 237-244.

[69] Tsyntsaru, N.; Kaziukaitis, G.; Yang, C.; Cesiulis, H.; Philipsen, H.G.G.; Lelis, M.; Celis, J.-P. Co-W nanocrystalline electrodeposits as barrier for interconnects. J. Solid State Electrochem., 2014, 18(11), 3057-3064.

[70] Kim, H.; Soper, S.A.; Podlaha, E.J. Pulse electrodeposition of multi-segmented super Invar/Au nanowires. ECS Transactions, 2013, 53(11), 9-14.

[71] Fukunaka, Y.; Motoyama, M.; Konishi, Y.; Ishii, R. Producing shape-controlled metal nanowires and nanotubes by an electrochemical method. Electrochem. Solid-State Lett., 2006, 9(3), C62C64.

[72] Cesiulis, H.; Xie, X.G.; Podlaha-Murphy, E. Electrodeposition of Co-W alloys with P and Ni. Mater. Sci. Medzg., 2009, 15(2), 115122.

[73] Motoyama, M.; Fukunaka, Y.; Ogata, Y.H.; Prinz, F.B. Impact of accompanying hydrogen generation on metal nanotube electrodeposition. J. Electrochem. Soc., 2010, 157(6), D357-D369.

[74] Davis, D.; Podlaha, E.J. CoNiCu and $\mathrm{Cu}$ nanotube electrodeposition. Electrochem. Solid St., 2005, 8(2), D1-D4. 
[75] Wang, Y.; Ye, C.; Changhui, F.; Xiaosheng, Z.; Zhang, L. A simple method for synthesizing copper nanotube arrays. Chem. Lett., 2004, 33(2), 166-167.

[76] Yoo, W.-C.; Lee, J.-K. Field-dependent growth patterns of metals electroplated in nanoporous alumina membranes. Adv. Mater., 2004, 16(13), 1097-1101.

[77] Philippe, L.; Michler, J. A kinetic model enabling controlled electrosynthesis of stacked metallic nanotubes and nanowires. Small, 2008, 4(7) 904-907.

[78] Song, C.; Wang, Z.; Chen, Q.; Cai, J.; Liu, L. High aspect ratio copper through-silicon-vias for 3D integration. Microelectron. Eng., 2008, 85(10), 1952-1956.

[79] Shin, S.; Kong, B.H.; Kim, B.S.; Kim, K.M.; Cho, H.K.; Cho, H.H. Over $95 \%$ of large-scale length uniformity in template-assisted electrodeposited nanowires by subzero-temperature electrodeposition. Nanoscale Res. Lett., 2011, 6(1), 467-474.

[80] Lopes, M.C.; de Oliveira, C.P.; Pereira, E.C. Computational modelling of the template-assisted deposition of nanowires. Electrochim. Acta, 2008, 53(13), 4359-4369.

[81] Reddy, S.M.; Park, J.J.; Na, S.M.; Maqableh, M.M.; Flatau, A.F.; Stadler, B.J.H. Electrochemical synthesis of magnetostrictive $\mathrm{Fe}-$ $\mathrm{Ga} / \mathrm{Cu}$ multilayered nanowire arrays with tailored magnetic response. Adv. Funct. Mater., 2011, 21(24), 4677-4683.

[82] Hyde, M.E.; Compton, R.G. How ultrasound influences the electrodeposition of metals. J. Electroanal. Chem., 2002, 531(1), 1924.

[83] Hamid, Z.A. Electrodeposition of cobalt-tungsten alloys from acidic bath containing cationic surfactants. Mater. Lett., 2003, 57(16-17), 2558-2564.

[84] Ramazani, A.; Almasi Kashi, M.; Alikhani, M.; Erfanifam, S. Fabrication of high aspect ratio Co nanowires with controlled magnetization direction using ac and pulse electrodeposition. Mater. Chem. Phys., 2008, 112(1), 285-289.

[85] Bade, K.; Leyendecker, K.; Thommes, A.; Bacher, W. In: Magnetic Materials, Processes and Device: Applications to Storage and Microelectromechanical systems (MEMS) In. Proc. of the $4^{\text {th }}$ Internat. Symp., Chicago, Ill., October 9-12, 1993. Romankiw, L.T., Ed.; Electrochemical Society: Pennington, N.J. 1996, 95, pp.697-708.

[86] Amatore, C.; Szunerits, S.; Thouin, L.; Warkocz, J.S. The real meaning of Nernst's steady diffusion layer concept under nonforced hydrodynamic conditions. A simple model based on Levich's seminal view of convection. J. Electroanal. Chem., 2001, 500(1-2), 62-70.

[87] Konishi, Y.; Motoyama, M.; Matsushima, H.; Fukunaka, Y.; Ishii, R.; Ito, $\mathrm{Y}$. Electrodeposition of $\mathrm{Cu}$ nanowire arrays with a template. J. Electroanal. Chem., 2003, 559(15), 149-153.

[88] Valizadeh, S.; George, J.M.; Leisner, P.; Hultman, L. Electrochemical deposition of Co nanowire arrays; quantitative consideration of concentration profiles. Electrochim. Acta, 2001, 47(6), 865874.

[89] Tsyntsaru, N.; Silkin, S.; Cesiulis, H.; Guerrero, M.; Pellicer, E.; Sort, J. Toward uniform electrodeposition of magnetic Co-W mesowires arrays: Direct versus pulse current deposition. Electrochim. Acta, 2016, 188, 589-601.

[90] Belevskii, S.S.; Cesiulis, H.; Tsyntsaru, N.I.; Dikusar, A.I. The role of mass transfer in the formation of the composition and structure of CoW coatings electrodeposited from citrate solutions. Surf. Eng. Appl. Electrochem., 2010, 46(6), 570-578.

[91] Blanco, S.; Vargas, R.; Mostany, J.; Borrás, C.; Scharifker, B.R. Modeling the growth of nanowire arrays in porous membrane. $J$. Electrochem. Soc., 2014, 161(8), E3341-E3347.

[92] Philippe, L.; Kacem, N.; Michler, J. Electrochemical deposition of metals inside high aspect ratio nanoelectrode array: Analytical current expression and multidimensional kinetic model for cobalt nanostructure synthesis. J. Phys. Chem. C, 2007, $111(13), 5229$ 5235 .

[93] Mulukutla, M.; Kommineni, V.K.; Harimkar, S.P. Pulsed electrodeposition of Co-W amorphous and crystalline coatings. Appl. Surf. Sci., 2012, 258(7), 2886-2893.

[94] Tsyntsaru, N.; Cesiulis, H.; Donten, M.; Sort, J.; Pellicer, E.; Podlaha-Murphy, E.J. Modern trends in tungsten alloys electrodeposi- tion with iron group metals. Surf. Eng. Appl. Electrochem., 2012, 48(6), 491-520.

[95] Ciureanu, M.; Beron, F.; Clime, L.; Ciureanu, P.; Yelon, A.; Ovari, T.A.; Cochrane, R.W.; Normandin, F.; Veres, T. Magnetic properties of electrodeposited $\mathrm{CoFeB}$ thin films and nanowire arrays. Electrochim. Acta, 2005, 50(22), 4487-4497.

[96] Cesiulis, H.; Tsyntsaru, N.; Ramanavicius, A.; Ragoisha, G. In: Nanostructures and Thin Films for Multifunctional Applications, Tiginyanu, I.; Topala, P.; Ursaki, V., Eds, Springer International Publishing Switzerland, 2016, pp. 3-42.

[97] Brenner, A. Electrodeposition of Alloys, ed.; Academic Press: New York, 1963.

[98] Landolt, D. Electrochemical and materials science aspects of alloy deposition. Electrochim. Acta, 1994, 39(8/9), 1075-1090.

[99] Llavona, Á.; Pérez, L.; Sánchez, M.C.; de Manuel, V. Enhancement of anomalous codeposition in the synthesis of $\mathrm{Fe}-\mathrm{Ni}$ alloys in nanopores. Electrochim. Acta, 2013, 106, 392-397.

[100] Matlosz, M. Competitive adsorption effects in the electrodeposition of iron-nickel alloys. J. Electrochem. Soc., 1993, 140(8), 22722279

[101] Zech, N.; Podlaha, E.J.; Landolt, D. Anomalous codeposition of iron group metals: II. mathematical model. J. Electrochem. Soc., 1999, 146(8), 2892-2900.

[102] Huang, Q.; Podlaha, E.J. Simulation of pulsed electrodeposition for GMR $\mathrm{FeCoNiCu} / \mathrm{Cu}$ multilayers. J. Electrochem. Soc., 2004, 151(2), C119-C126.

[103] Dragos, O.; Chiriac, H.; Lupu, N.; Grigoras, M.; Tabakovic, I. Anomalous codeposition of fcc NiFe nanowires with $5-55 \% \mathrm{Fe}$ and their morphology, crystal structure and magnetic properties. $J$ Electrochem. Soc., 2016, 163(3), D83-D94.

[104] Atalay, F.E.; Kaya, H.; Atalay, S.; Tari, S. Influences of deposition time and $\mathrm{pH}$ on magnetic NiFe nanowires fabrication. J. Alloys Compd., 2009, 469(1), 458-463.

[105] Dahms, H.; Croll, I.M. The anomalous codeposition of iron-nickel alloys. J. Electrochem. Soc., 1965, 112(8), 771-775.

[106] Zhu, H.; Yang, S.; Ni, G.; Yu, D.; Du, Y. Fabrication and magnetic properties of $\mathrm{Co}_{67} \mathrm{Ni}_{33}$ alloy nanowire array. Scripta Mater., 2001, 44(8-9), 2291-2295.

[107] Vilana, J.; Gómez, E.; Vallés, E. Electrochemical control of composition and crystalline structure of CoNi nanowires and films prepared potentiostatically from a single bath. J. Electroanal. Chem., 2013, 703, 88-96.

[108] Zhan, Q.; Chen, Z.; Xue, D.; Li, F.; Kunkel, H.; Zhou, X.; Roshko, R.; Williams, G. Structure and magnetic properties of $\mathrm{Fe}-\mathrm{Co}$ nanowires in self-assembled arrays. Phys. Rev. B, 2002, 66(13), 134436.

[109] Elbaile, L.; Crespo, R.D.; Vega, V.; García, J.A.; Garcia, J.A. Magnetostatic interaction in Fe-Co nanowires. J. Nanomater., 2012, 2012, 1-6.

[110] Khan, H.R.; Petrikowski, K. Magnetic and structural properties of the electrochemically deposited arrays of $\mathrm{Co}$ and CoFe nanowires. J. Magn. Magn. Mater., 2002, 249(3), 458-461.

[111] Han, G.; Lu, J.; Gao, Y. FeCo nanowires deposited in a magnetic field. J. Magn. Magn. Mater., 2015, 393, 199-203.

[112] Song, Y.; Lu, W,; Xu, Y.; Shi, J.; Fang, X. Growth of singlecrystalline $\mathrm{Co}_{7} \mathrm{Fe}_{3}$ nanowires via electrochemical deposition and their magnetic properties. J. Alloys Compd., 2015, 652, 179-184.

[113] Huang, Q.; Davis, D.; Podlaha, E.J. Electrodeposition of FeCoNiCu nanowires. J. Appl. Electrochem., 2006, 36(8), 871-882.

[114] Geng, X.; Podlaha, E.J. Coupled, simultaneous displacement and dealloying reactions into $\mathrm{Fe}-\mathrm{Ni}-\mathrm{Co}$ nanowires for thinning nanowire segments. Nano Lett., 2016, 16(12), 7439-7445.

[115] Li, D.; Podlaha, E.J. Template Fe-Ni-Co nanowire electrodeposition with $\mathrm{H}_{2}$ evolving side reactions. ECS Meeting Abstract, 2016, MA2016-01(41), 2073.

[116] Samanifar, S.; Almasi Kashi, M.; Ramazani, A.; Alikhani, M. Reversal modes in FeCoNi nanowire arrays: Correlation between magnetostatic interactions and nanowires length. J. Magn. Magn. Mater., 2015, 378, 73-83.

[117] Saedi, A.; Ghorbani, M. Electrodeposition of Ni-Fe-Co alloy nanowire in modified AAO template. Mater. Chem. Phys., 2005, 91(2), 417-423. 
[118] Stojic, D.L.; Marceta, M.P.; Sovilj, S.P.; Miljanic, S.S. Hydrogen generation from water electrolysis - possibilities of energy saving. J. Power Sources, 2003, 118(1-2), 315-319.

[119] Anantharaj, S.; Ede, S.R.; Sakthikumar, K.; Karthick, K.; Mishra, S.; Kundu, S. Recent trends and perspectives in electrochemical water splitting with an emphasis on sulfide, selenide, and phosphide catalysts of $\mathrm{Fe}, \mathrm{Co}$, and Ni: A review. ACS Catal., 2016, 6(12), 8069-8097.

[120] Lee, H.-B.; Tsau, J.-C.; Lee, C.-Y. HER catalytic activity of electrodeposited Ni-P nanowires under the influence of magnetic field. J. Nanomater., 2013, 2013, Article ID 191728.

[121] Lu, G.; Evans, P.; Zangari, G. Electrocatalytic properties of Nibased alloys toward hydrogen evolution reaction in acid media. $J$. Electrochem. Soc., 2003, 150(5), A551-A557.

[122] Krolikowski, A.; Wiecko, A. Impedance studies of hydrogen evolution on Ni-P alloys. Electrochim. Acta, 2002, 47(13-14), 20652069.

[123] Lee, H.-B.; Hsu, C.-H.; Wu, D.-S. A study on the hydrogen evolving activity of electroplated Ni-P coating by using the taguchi method. J. New Mat. Electrochem. Syst., 2011, 14(4), 237-245.

[124] Chen, P.-C.; Chang, Y.-M.; Wu, p.-W.; Chiu, Y.-F. Fabrication of Ni nanowires for hydrogen evolution reaction in a neutral electrolyte. Int. J. Hydrogen Energy, 2009, 34(16), 6596-6602.

[125] Iida, T.; Matsushima, H.; Fukunaka, Y. Water electrolysis under a magnetic field. J. Electrochem. Soc., 2007, 154(8), E112-E115.

[126] Matsushima, H.; Kiuchi, D.; Fukunaka, Y. Measurement of dissolved hydrogen supersaturation during water electrolysis in a magnetic field. Electrochim. Acta, 2009, 54(24), 5858-5862.

[127] Ezaki, H.; Morinaga, M.; Watanabe, S.; Saito, J. Hydrogen overpotential for intermetallic compounds, $\mathrm{TiAl}, \mathrm{FeAl}$ and NiAl, containing 3d transitionmetals. Electrochim. Acta, 1994, 39(11-12), 17691773.

[128] Burchardt, T.; Hansen, V.; Valand, T. Microstructure and catalytic activity towards the hydrogen evolution reaction of electrodeposited NiPx alloys. Electrochim. Acta, 2001, 46(18), 2761-2766.

[129] Paseka, I. Evolution of hydrogen and its sorption on remarkable active amorphous smooth $\mathrm{NiP}(\mathrm{x})$ electrodes. Electrochim. Acta, 1995, 40(11), 1633-1640.

[130] Shervedani, R.K.; Lasia, A. Studies of the hydrogen evolution reaction on Ni-P electrodes. J. Electrochem. Soc., 1997, 144(2), 511-519.

[131] Hsu, S.; Lee, C. H.B.; Lin, C.S.; Lee, C.Y. The electrocatalytic activity of electrodeposited Ni-P micro-patterned structure in acidic solution. J. Chin. Soc. Mechan. Eng., 2011, 32, 103-109.

[132] Liu, T.; Liu, Q.; Asiri, A.M.; Luo, Y.; Sun, X. An amorphous CoSe film behaves as an active and stable full water-splitting electrocatalyst under strongly alkaline conditions. Chem. Commun., 2015, 51(93), 16683-16686.

[133] Carim, A.I.; Saadi, F.H.; Soriaga, M.P.; Lewis, N.S. Electrocatalysis of the hydrogen-evolution reaction by electrodeposited amorphous cobalt selenide films. J. Mater. Chem. A, 2014, 2(34), 13835-13839.

[134] He, L.; Qin, L.; Zhao, J.; Yang, Y.; Yin, Y. Preparation of Pt/Ni multilayer nanowires with enhanced magnetic property and electrocatalytic activity. J. Nano Res., 2016, 40, 20-28.

[135] Bertin, E.; Garbarino, S.; Ponrouch, A.; Guay, D. Synthesis and characterization of PtCo nanowires for the electro-oxidation of methanol. J. Power Sources, 2012, 206, 20-28.

[136] Vernickaite, E.; Tsyntsaru, N.; Cesiulis, H. Electrochemical codeposition of tungsten with cobalt and copper: Peculiarities of binary and ternary alloys coatings formation. Surf. Coat. Tech., 2016, 307(Part C), 1341-1349.

[137] Piraux, L.; Dubois, S.; Duvail, J.L.; Ounadjela, K.; Fert, A. Arrays of nanowires of magnetic metals and multilayers: Perpendicular GMR and magnetic properties. J. Magn. Magn. Mater., 1997, 175(1-2), 127-136.

[138] Özkale, B.; Shamsudhin, N.; Chatzipirpiridis, G.; Hoop, M.; Gramm, F.; Chen, X.; Martí, X.; Sort, J.; Pellicer, E.; Pané, S. Multisegmented $\mathrm{FeCo} / \mathrm{Cu}$ nanowires: Electrosynthesis, characterization, and magnetic control of biomolecule desorption. ACS Appl. Mater. Interfaces, 2015, 7(13), 7389-7396.
[139] Zhang, M.J.; Agramunt-Puig, S.; Del-Valle, N.; Navau, C.; Baró, M.D.; Estradé, S.; Peiró, F.; Pané, S.; Nelson, B.J.; Sanchez, A.; Nogués, J.; Pellicer, E.; Sort, J. Tailoring staircase-like hysteresis loops in electrodeposited trisegmented magnetic nanowires: A strategy toward minimization of interwire interactions. ACS Appl. Mater. Interfaces, 2016, 8(6), 4109-4117.

[140] Zhang, J.; Pané, S.; Sort, J.; Pellicer, E. Toward robust segmented nanowires: Understanding the impact of crystallographic texture on the quality of segment interfaces in magnetic metallic nanowires. Adv. Mater. Interfaces, 2016, 3(18), 1600336.

[141] Zeng, M.H.; Skomski, R.; Menon, L.; Liu, Y.; Bandyopadhyay, S.; Sellmyer, D.J. Structure and magnetic properties of ferromagnetic nanowires in self-assembled arrays. Phys. Rev. B, 2002, 65, 134426 ,

[142] Cattaneo, L.; Franz, S.; Albertini, F.; Ranzieri, P.; Vicenzo, A.; Bestetti, M.; Cavallotti, P.L. Electrodeposition of hexagonal Co nanowires with large magnetocrystalline anisotropy. Electrochim. Acta, 2012, 85, 57-65.

[143] Irshad, M.I.; Mohamed, N.M.; Abdullah, M.Z.; Saheed, M.S.M.; Mumtaz, A.; Yasar, M.; Yar, A.; Zeeshan, M.A.; Sort, J. Influence of the electrodeposition potential on the crystallographic structure and effective magnetic easy axis of cobalt nanowires. RSC Adv., 2016, 6, 14266.

[144] Thongmee, S.; Pang, H.L.; Yi, J.B.; Ding, J.; Lin, J.Y.; Van, L.H. The structure and magnetic properties of metal and alloy nanowires via AAO template. Int. J. Nanosci., 2009, 8(1-2), 75-80.

[145] Jo, C.; Lee, J.I.; Jang, Y. Electronic and magnetic properties of ultrathin Fe-Co alloy nanowires. Chem. Mater., 2005, 17(10), 2667-2671.

[146] Zhang, X.; Zhang, H.; Wu, T.; Li, Z.; Zhang, Z.; Sun, H. Comparative study in fabrication and magnetic properties of $\mathrm{FeNi}$ alloy nanowires and nanotubes. J. Magn. Magn. Mater., 2013, 331, 162167.

[147] Almasi Kashi, M.; Ramazani, A.; Asgari, V.; Jafari-Khamse, E. Magnetic properties of $\mathrm{Ni}_{0.3} \mathrm{Fe}_{0.7}$ alloy nanowires. J. Nanostruct., 2013, 3(1), 11-15.

[148] Wang, M.Y.W.; Zhang, L.D.; Meng, G.W.; Peng, X.S.; Jin, Y.X.; Zhang, J. Fabrication of ordered ferromagnetic-nonmagnetic alloy nanowire arrays and their magnetic property dependence on annealing temperature. J. Phys. Chem. B, 2002, 106(10), 2502-2507.

[149] Hao, Z.; Shaoguang, Y.; Gang, N.; Dongliang, Y.; Youwei, D. Study on magnetic property of $\mathrm{Fe}_{14} \mathrm{Ni}_{86}$ alloy nanowire array. $J$. Magn. Magn. Mater., 2001, 234(3), 454-458.

[150] Ghemes, A.; Dragos-Pinzaru, O.; Chiriac, H.; Lupu, N.; Grigoras, M.; Shore, D.; Stadler, B.; Tabakovic, I. Controlled electrodeposition and magnetic properties of $\mathrm{Co}_{35} \mathrm{Fe}_{65}$ nanowires with high saturation magnetization. J. Electrochem. Soc., 2017, 164(2), D13-D22.

[151] Koohbor, M.; Soltanian, S.; Najafi, M.; Servati, P. Fabrication of CoZn alloy nanowire arrays: Significant improvement in magnetic properties by annealing process. Mater. Chem. Phys., 2012, 131(3), 728-734.

[152] Qin, D.H.; Cao, L.; Sun, Q.Y.; Huang, Y.; Li, H.L. Fine magnetic properties obtained in FeCo alloy nanowire arrays. Chem. Phys. Lett., 2002, 358(5-6), 484-488.

[153] Qin, D.H.; Zhang, H.L.; Xu, C.L.; Xu, T.; Li, H.L. Magnetic domain structure in small diameter magnetic nanowire arrays. Appl. Surf. Sci., 2005, 239(3-4), 279-284.

[154] Najafi, M.; Rafati, A.A.; Fart, M.K.; Zare, A. Effect of the pH and electrodeposition frequency on magnetic properties of binary Co1xSnx nanowire arrays. J. Mater. Res., 2014, 29(2), 190-196.

[155] Yuan, X.Y.; Wu, G.S.; Xie, T.; Lin, Y.; Meng, G.W.; Zhang, L.D. Autocatalytic redox fabrication and magnetic studies of $\mathrm{Co}-\mathrm{Ni}-\mathrm{P}$ alloy nanowire arrays. Solid State Commun., 2004, 130(6), 429432.

[156] Salazar-Alvarez, G.; Geshev, J.; Agramunt-Puig, S.; Navau, C.; Sanchez, A.; Sort, J.; Nogués, J. Tunable high-field magnetization in strongly exchange-coupled freestanding $\mathrm{Co} / \mathrm{CoO}$ Core/Shell Coaxial nanowires. ACS App. Mater. Interfaces, 2016, 8(34), 2247722483.

[157] Maurice, J.-L.; Imhoff, D.; Etienne, P.; Durand, O.; Dubois, S.; Piraux, L.; George, J.-M.; Galtier, P.; Fert, A. Microstructure of 
magnetic metallic superlattices grown by electrodeposition in membrane nanopores. J. Magn. Magn. Mater., 1998, 184(1), 1-18.

[158] Serrà, A.; Alcobé, X.; Sort, J.; Nogués, J.; Vallés, E. Highly efficient electrochemical and chemical hydrogenation of 4-nitrophenol using recyclable narrow mesoporous magnetic CoPt nanowires. $J$. Mater. Chem. A, 2016, 4(40), 15676-15687.

[159] Davis, D.M.; Moldovan, M.; Young, D.P.; Henk, M.; Xie, X.; Podlaha, E.J. Magnetoresistance in electrodeposited CoNiFeICu multilayered nanotubes. Electrochem. Solid-State Lett., 2006, 9(9), C153-C155.

[160] Vázquez, M.; Pirota, K.; Torrejo, J.; Navas, D.; Hernandez-Velez, M. Magnetic behaviour of densely packed hexagonal arrays of $\mathrm{Ni}$ nanowires: Influence of geometric characteristics. J. Magn. Magn. Mater., 2005, 294(2), 174-181.

[161] Ivanov, Y.P.; Vázquez, M.; Chubykalo-Fesenko, O. Magnetic reversal modes in cylindrical nanowires. J. Phys. D. Appl. Phys., 2013, 46, Article No. 485001

[162] Vivas, G.L.; Vázquez, M.; Escrig, J.; Allende, S.; Altbir, D.; Leitao, D.C.; Araujo, J.P. Magnetic anisotropy in CoNi nanowire arrays: Analytical calculations and experiments. Phys. Rev. B, 2012, 85,035439

[163] Sellmyer, D.J.; Zheng, M.; Skomski, R. Magnetism of Fe, Co and Ni nanowires in self-assembled arrays. J. Phys. Condens. Matter., 2001, 13, R433-R460.

[164] Rivas, J.; Bantu, A.K.M.; Zaragoza, G.; Blanco, M.C.; LópezQuintela, M.A. Preparation and magnetic behavior of arrays of electrodeposited Co nanowires. J. Magn. Magn. Mater., 2002, 249(1-2), 220-227.

[165] Vázquez, M.; Vivas, L.G. Magnetization reversal in Co-base nanowire arrays. Phys. Status Solidi B, 2011, 248(10), 2368-2381.

[166] McGary, P.D.; Tan, L.W.; Zou, J.; Stadler, B.J.H.; Downey, P.R.; Flatau, A.B. Magnetic nanowires for acoustic sensors. J. Appl. Phys., 2006, $99(8), 308-310$

[167] Kartopu, G.; Yalçın, O. Fabrication and applications of metal nanowire arrays electrodeposited in ordered porous templates. In Electrodeposited Nanowires and their Applications Ed. Lupu, N. Publisher InTech; 2010, pp.113-140.

[168] Bakonyi, I.; Péter, L. Electrodeposited multilayer films with giant magnetoresistance (GMR): Progress and problems. Prog. Mater Sci., 2010, 55(3), 107-245.

[169] Alper, M.; Attenborough, K.; Hart, R.; Lane, S.J.; Lashmore, D.S.; Younes, C.; Schwarzacher, W. Giant magnetoresistance in electrodeposited superlattices. Appl. Phys. Lett., 1993, 63(15), 2144 2146.

[170] Evans, P.R.; Yi, G.; Schwarzacher, W. Current perpendicular to plane giant magnetoresistance of multilayered nanowires electrodeposited in anodic aluminum oxide membranes. Appl. Phys. Lett., 2000, 76(4), 481-483.

[171] Davis, D.; Zamanpour, M.; Moldovan, M.; Young, D.; Podlaha, E.J. Electrodeposited, GMR $\mathrm{CoNiFeCu}$ nanowires and nanotubes from electrolytes maintained at different temperatures. J. Electrochem. Soc., 2010, 157(6), D317-D322. 